\title{
Determining Heterogeneous Slip Activity on Multiple Slip Systems from Single Crystal Orientation Pole Figures
}

\author{
Darren C. Pagan ${ }^{1}$ and Matthew P. Miller ${ }^{2,3}$ \\ ${ }^{1}$ Lawrence Livermore National Laboratory \\ ${ }^{2}$ Sibley School Of Mechanical and Aerospace Engineering, Cornell University \\ ${ }^{3}$ Cornell High Energy Synchrotron Source
}

June 5, 2016

\begin{abstract}
A new experimental method to determine heterogeneity of shear strains associated with crystallographic slip in the bulk of ductile, crystalline materials is outlined. The method quantifies the time resolved evolution of misorientation within plastically deforming crystals using single crystal orientation pole figures (SCPFs) measured in-situ with X-ray diffraction. A multiplicative decomposition of the crystal kinematics is used to interpret the distributions of lattice plane orientation observed on the SCPFs in terms of heterogeneous slip activity (shear strains) on multiple slip systems. To show the method's utility, the evolution of heterogeneous slip is quantified in a silicon single crystal plastically deformed at high temperature at multiple load steps, with slip activity in sub-volumes of the crystal analyzed simultaneously.
\end{abstract}

\section{Introduction}

Crystallographic slip is the primary mode of plastic deformation in ductile, crystalline materials (notably engineering alloys): under application of shear stresses, dislocations move on particular crystallographic planes in prescribed directions. Crystallographic slip remains an important area of research. There are many important material failure modes, such as yielding, fatigue, and ductile fracture, that have been directly linked to slip. Often it is the heterogeneity of slip, the formation of intracrystal regions that experience different slip modes or the localization of slip, that appears to matter most for material failure. An accurate, detailed understanding of slip processes and the manifestation of slip-induced material evolution therefore has the potential for significant impact over a broad range of materials topics and applications. Modern micromechanical models of the plastic behavior of crystalline materials are built to accommodate representations of slip, but a detailed understanding of the complexities of the slip initiation 
process, driven by the subgrain mechanical environment, has proven illusive. Like many deformation processes, in-situ experimental observations of slip inside deforming crystals are difficult to acquire and most of what we know about slip has been inferred from post-mortem micrographs. Measurements of slip traces $[1,2]$ and dislocation structures using electron microscopy $[3,4]$ have helped to further our understanding of slip processes, but if we wish to fully model and predict plasticity driven failure processes, our experimental measures of slip must improve. We need to be able to quantitatively measure the complicated dynamics of slip processes within the bulk of samples to capture the initiation and acceleration of failure processes. Therefore, we need probes with rapid collection times and significant penetration depth used in conjunction with models to interpret these raw data in terms of crystallographic slip. To help meet this need, this paper presents a new experimental method which uses X-ray diffraction data measured at a synchrotron source with a kinematic model of crystallographic slip to quantify the heterogeneity of shear strains on different slip systems within ductile crystals as they plastically deform. Our method relates the heterogeneity observed in distributions of diffracted X-ray intensity to the variation of rotation of a set of crystallographic planes and the underlying cause, plastic slip on a particular slip system. The method is capable of probing slip during in-situ mechanical loading in sub-volumes of crystal that are deforming using different combinations of slip systems.

There is a long history of using X-ray diffraction to study crystallographic slip. Slip was inferred from deformation-induced changes in the patterns of diffracted X-ray intensity [5, 6], and in fact served as motivation for long-lasting theories of slip and dislocation motion [7]. Typically when studying slip with X-ray diffraction, models are necessary to relate slip activity to quantities that are measurable through diffraction processes, such as elastic strain or lattice orientation. Recent efforts studying heterogeneous slip activity within individual crystals generally rely on using models to relate diffracted intensity to the density fields of dislocations present in the crystal. Distributions of elastic strain $[8,9,10]$ or misorientation $[11,12]$ produced by the dislocation density fields are measured and subsequently used to determine the dislocation density, dislocation type, and active slip systems from the diffraction data. Approaches to understanding slip through analysis of distributions of lattice strain or orientation each have their own benefits. Strain based methods are sensitive to both statistically stored (SSD) and geometrically necessary (GND) dislocation types, making them well-suited for understanding the development of material strength. A downside of this approach is that there is typically insufficient instrument 
resolution to associate distributions of strain to sub-volumes of the region probed when analyzing line profile data (intensity versus $2 \theta$ ). Unlike distributions of strain, distributions of lattice orientation are only sensitive to GND content present in the diffraction volume. However, subvolumes deforming by different slip systems will have marked differences in GND content and if sufficiently misoriented, can be identified and isolated in distributions of lattice orientation. The ability to differentiate sub-volumes of crystal makes orientation based methods attractive for studying heterogeneity of slip at sub-crystal length scales, and subsequently, the precursors to material failure.

With the increased use of modern synchrotron sources for studying the mechanical deformation of engineering materials, X-ray experiments to study slip processes are becoming increasingly important. These sources, with their ability to provide high-energy X-ray beams at enormous flux, combined with large, fast area detectors are enabling rapid measurements of complete three dimensional distributions of diffracted intensity. These new diffraction data, along with in-situ sample environments are enabling a new generation of mechanical tests in which the evolution of quantities associated with plastic deformation, including distributions of elastic strain $[13,14,15]$ and crystal orientation $[16,17,18]$, can be studied with significantly finer temporal and spatial resolution $[19,20]$. New experiments and data processing models designed for use at synchrotron sources promise to advance our capability to experimentally measure slip processes and improve our understanding of crystal plasticity in manners not possible using optical or electron microscopy methods.

In this paper, we use the enormous quantity of diffracted intensity data that is possible to acquire at a synchrotron light-source, along with a kinematic model of crystallographic slip, to correlate measured single crystal pole figures with slip system activity. A single pole figure only provides information about the orientation distribution from a set of lattice planes, but from multiple pole figures, heterogeneity of slip can be quantified. We present experimental results from a single crystal silicon compression specimen deformed at high temperature, with silicon serving as a model material for other materials, including metals. In $\S 2$, an introduction to single crystal pole figures and how their basic features relate to misorientation distributions within a crystal is given. A kinematic model is introduced to relate the evolution of single crystal pole figures to heterogeneity of slip. Then a description of the experiment geometry and the process to produce single crystal orientation pole figures from area detector diffraction data is in $\S 4$. $\S 5$ 
presents single crystal pole figures measured in-situ from a plastically deforming silicon single

crystal and the evolution of lattice misorientation as the crystal deformed. In $§ 5.1$ the slip model is used to determine heterogeneous slip activity from the deforming silicon crystal. In $\S 6$, the slip activity results are discussed and extension of the presented experimental method to the study of grains in polycrystals is described.

In this paper, bold characters will indicate vectors and tensors $(\boldsymbol{a}, \boldsymbol{A})$. A vector in the undeformed configuration is denoted with an overbar $\overline{\boldsymbol{a}}$. The vectors $\boldsymbol{e}_{\boldsymbol{x}}, \boldsymbol{e}_{\boldsymbol{y}}$, and $\boldsymbol{e}_{\boldsymbol{z}}$ will be reserved for basis vectors of rectangular Cartesian coordinate systems. Superscripts will denote the coordinate systems in which vectors or tensors are expressed $\left(\boldsymbol{a}^{S}\right)$, except $\mathrm{T}$ reserved for a transpose operation. The superscripts S, L, C, and DET denote the sample, laboratory, crystal, and detector coordinate systems respectively. Subscripts will generally identify specific scalars, vectors or tensors $\left(a_{\mathbf{0}}, \boldsymbol{a}_{\mathbf{0}}, \boldsymbol{A}_{\mathbf{0}}\right)$. The dot product of a second order tensor and a vector is $\boldsymbol{A} \cdot \boldsymbol{b}$ $\left(A_{i j} b_{j}\right)$, while the dot product of two second order tensors) is defined as $\boldsymbol{A} \cdot \boldsymbol{B}\left(A_{i j} B_{j k}\right)$. The vector outer product is defined as $\boldsymbol{a} \otimes \boldsymbol{b}\left(a_{i} b_{j}\right)$. The operation of building a skew-symmetric matrix from a vector is defined as $\boldsymbol{a} \times \boldsymbol{I}\left(\epsilon_{i k j} a_{k}\right.$ where $\epsilon$ is the permutation symbol).

\section{Single Crystal Orientation Pole Figures and Distributions of Orienta- tion}

Distributions of lattice orientation within individual crystals are a by-product of slip processes, so through measurement of these distributions and their evolution, we can infer information about the underlying slip processes. The measurement of distributions of lattice plane normals with respect to an external coordinate system, or pole figures, is one of the primary tools for quantifying lattice plane orientation non-destructively in crystalline materials [21]. Building a pole figure for a particular family of lattice planes (denoted by Miller indices $\{h k l\}$ ) begins with determining the intersection of the plane normal $\boldsymbol{n}$ (a pole) with a unit sphere. The sphere represents every possible plane orientation with respect to an external coordinate system. Fields expressed over a pole figure indicate the presence of a plane in a particular orientation or a physical quantity, for instance, lattice strains [22, 23, 24]. A pole figure associated with a family of lattice planes $\{h k l\}\left(P_{\{h k l\}}\right)$ is most commonly used to indicate the probability of finding 
lattice planes in a particular orientation [25, 21], and has also been used to find orientation distributions in single crystals $[26,27]$. There is a close connection between orientation pole figure data and X-ray diffraction measurements: every value on a pole figure represents a crystal volume containing lattice planes with normal $\boldsymbol{n}$, and each crystal volume containing lattice planes with that normal will contribute to the measured diffracted intensity.

In contrast to traditional studies of textures in fine-grained polycrystalline samples, when diffraction peaks from individual crystals can be resolved, pole figures can be generated for specific sets of lattice planes, rather then symmetrically related families. On these single crystal orientation pole figures (SCPFs), plane normal densities are zero for most possible lattice plane orientations (see Figure 1). As such, most of the SCPF can be neglected and focus is placed on non-zero values clustered within a few degrees of the average plane orientation. In this work, the small region of interest is shown with an aerial view of the pole figure. Figure 1 illustrates a more familiar complete pole figure and a magnification of the region of interest as an example to acclimate the reader to the data presented. From this point onward, only SCPFs are discussed, so the parentheses indicating a specific set of lattice planes, rather than a family of lattice planes will no longer be included $\left(P_{(h k l)}=P_{h k l}\right)$.

When studying crystallographic texture using pole figures, all spatial information about the position of a grain with a specific orientation is lost. This is also true for SCPFs: the spatial arrangement of probed crystal volumes does not influence the SCPF and only the angular distribution of lattice plane orientation is measured. However, if some degree of lattice continuity is assumed [7], reasonable assumptions can be made about the arrangement of misoriented volumes within a single crystal or grain. Examples are presented tying spatial distributions of misorientation to SCPFs in order to aid interpretation of data presented. If different volumes of crystal are misoriented relative to one another about a single misorientation axis $\boldsymbol{t}$, the normals of a set of lattice planes $(h k l)$ from these volumes lie on a common arc on the SCPF. This effect is shown in Figure 2. In the Figure, three volumes of crystal (with $\boldsymbol{n}$ labeled 1-3) are rotated relative to one another by varying amounts about the axis, $\boldsymbol{t}$. In the Figure, $\boldsymbol{t}$ is the [001] crystallographic direction and the lattice planes shown are the (010) set of lattice planes.

To quantify the misorientation of lattice planes on a SCPF, the vector $\boldsymbol{v}$ is introduced. This vector describes the extension of a pole figure along a trajectory. While the distributions of plane normals spread along arcs, if these arcs are small relative to the circumference of the sphere, 
they can be approximated as vectors (as is the case when misorientation is of the order of a few degrees). An example of the vector $\boldsymbol{v}$ on a SCPF has been illustrated for the distribution of lattice plane orientation in Figure 2. The vector $\boldsymbol{v}$ describing the trajectory of extension is related to the maximum misorientation present across a crystal volume $\Delta \phi$, the axis $\boldsymbol{t}$, and the reference plane normal position of a set of lattice planes $\boldsymbol{n}$ :

$$
\boldsymbol{v}=\Delta \phi \boldsymbol{t} \times \overline{\boldsymbol{n}}=\boldsymbol{w} \times \overline{\boldsymbol{n}}
$$

where we define a misorientation vector $\boldsymbol{w}$ as $\Delta \phi \boldsymbol{t}$. A complete derivation of Equation 1 can be found in Appendix A. From this equation, we find that for the same $\Delta \phi, \boldsymbol{v}$ corresponding to lattice planes with normals $\boldsymbol{n}$ that are $90^{\circ}$ away from $\boldsymbol{t}$ (and $\boldsymbol{w}$ ) have the largest magnitude, while $\boldsymbol{v}$ is equal to $\mathbf{0}$ when $\boldsymbol{t}$ is parallel to $\overline{\boldsymbol{n}}$. A trajectory $\boldsymbol{v}$ quantifies the misorientation of a set of lattice planes, and from $\boldsymbol{v}$ measured from multiple sets of lattice planes, the misorientation of the crystal lattice can be found. In the example, discrete lattice plane normals are shown to make clear how plane normals are shifted relative to one another on the sphere as a crystal develops misorientation about an axis. In real experimental data, the lattice planes within an interrogated crystal volume often contribute to continuous distributions, but $\boldsymbol{v}$ can also be readily measured from these continuous distributions, and the relationship between $\boldsymbol{v}$ and the misorientation axis $\boldsymbol{t}$ is still the same.

Continuing further, sub-volumes of a crystal may develop misorientation about different axes. The different misorientation axes present in the crystal are $\boldsymbol{t}_{\boldsymbol{\beta}}$ where $\beta$ indicates a specific axis. An example of this type of misorientation distribution with two distinct axes is shown in Figure 3 (noting that more than two misorientation axes are possible). In Figure 3, the (010) lattice planes from the three crystal volumes are again misoriented about $\boldsymbol{t}=[001]$, however, the first crystal volume has also developed misorientation about a second distinct axis (the [100] crystallographic direction). The sub-divisions of volume 1 are labeled $1 \mathrm{a}, 1 \mathrm{~b}$, and $1 \mathrm{c}$. The initial misorientation axis (the [001] crystallographic direction) has been re-labeled $\boldsymbol{t}_{\mathbf{1}}$, while the new misorientation axis is labeled $\boldsymbol{t}_{\mathbf{2}}$. The corresponding pole figure, $P_{010}$, is shown to the right. With this misorientation configuration, two distinct streaking directions, $\boldsymbol{v}_{\mathbf{1}}$ and $\boldsymbol{v}_{\mathbf{2}}$, are discernible on each SCPF.

Two distinct types of heterogeneity of reorientation have now been defined. First, within a 
crystal, volumes can be reorienting at different rates about a common axis $\boldsymbol{t}$. Second, multiple distinct sub-volumes of crystal can be reorienting about different axes $\boldsymbol{t}_{\boldsymbol{\beta}}$. The first type of misorientation manifests as spreading along a trajectory $\boldsymbol{v}$ on a SCPF, and the second type of misorientation manifests as multiple $\boldsymbol{v}_{\boldsymbol{\beta}}$ existing on the SCPF. If no distinct misorientation axes are present, the distribution of lattice plane orientation will not extend along well-defined trajectories $\boldsymbol{v}$.

\section{Relating the Kinematics of Slip to Single Crystal Pole Figure Data}

The trajectories $\boldsymbol{v}$ on a SCPF and the misorientation vector $\boldsymbol{w}$ will be related to the heterogeneity of slip activity during loading of single crystals using a multiplicative decomposition of the kinematics of a deforming crystal. We aim to take information about distributions of lattice plane orientation and from these data, quantify the heterogeneity of slip. The deformation of single crystals in compression will be described, but a similar analysis can be performed for single crystals deformed in tension. We emphasize that the goal of this model is not to serve as a predictive model for material deformation, but rather to extract quantitative mechanical response data in a specific deformation regime.

In an elasto-viscoplastic description of a crystal plastically deforming by slip, the deformation can be described as a simple shearing of a volume of crystal due crystallographic slip, followed by a rotation and stretch of the volume of crystal. The shearing of the crystal volume leaves the lattice unchanged, while the subsequent rotation and stretch alters the periodicity of the lattice. The deformation is described by a multiplicative decomposition of the deformation gradient $\boldsymbol{F}$ and is approximated as [28]

$$
\boldsymbol{F}=\boldsymbol{F}^{*} \cdot \boldsymbol{F}_{\boldsymbol{P}}
$$

where $\boldsymbol{F}_{\boldsymbol{P}}$ is the portion of the deformation due to slip and $\boldsymbol{F}^{*}$ is the rotation and stretch of the material and lattice. If elasticity is neglected, $\boldsymbol{F}^{*}$ consists solely of a rotation $\boldsymbol{R}^{*}$. The simple shearing motion $\boldsymbol{F}_{\boldsymbol{P}}$ is further decomposed into a rotation, $\boldsymbol{R}_{\boldsymbol{P}}$, and a right symmetric stretch, $U_{P}$

$$
\boldsymbol{F}=\boldsymbol{R}^{*} \cdot \boldsymbol{R}_{P} \cdot \boldsymbol{U}_{P}=\boldsymbol{R} \cdot \boldsymbol{U}_{\boldsymbol{P}}
$$

where $\boldsymbol{R}$ is defined as the total rotation of material [29] and the determinant of $\boldsymbol{F}$ is 1 (the 
deformation is isochoric). Importantly, only the portions of the deformation that alter the spacing and orientation of the lattice can be measured using diffraction. As such, only $\boldsymbol{R}^{*}$ (or $\left.\boldsymbol{F}^{*}\right)$ is a measurable quantity and it is equal to [30]

$$
\boldsymbol{R}^{*}=\boldsymbol{R} \cdot \boldsymbol{R}_{\boldsymbol{P}}^{T}
$$

When a crystal undergoes large plastic deformations, it is not possible to decompose the plastic portion of the deformation gradient, $\boldsymbol{F}_{\boldsymbol{P}}$, into shearing motions on individual slip systems. However, for the experimental data to be analyzed, the single crystal deformation extends just beyond the elastic-plastic transition and is assumed to be small. In this regime, the total plastic deformation can be additively decomposed on to each slip system, and $\boldsymbol{F}_{\boldsymbol{P}}$ is approximated as a linear combination of shears on different slip systems:

$$
\boldsymbol{F}_{\boldsymbol{P}}=\boldsymbol{I}+\sum_{\alpha=1}^{\mathrm{SS}} \gamma_{\alpha}\left(\overline{\boldsymbol{s}}_{\boldsymbol{\alpha}} \otimes \overline{\boldsymbol{n}}_{\boldsymbol{\alpha}}\right)
$$

where $\alpha$ denotes a specific slip system, $\gamma_{\alpha}$ is the total shear strain on a slip system, $\boldsymbol{s}_{\boldsymbol{\alpha}}$ is the slip direction, $\boldsymbol{n}_{\boldsymbol{\alpha}}$ is the slip plane normal of each slip system, and $\overline{\boldsymbol{s}}_{\boldsymbol{\alpha}} \otimes \overline{\boldsymbol{n}}_{\boldsymbol{\alpha}}$ is the Schmid tensor. The combination of a finite number of possible simple shearing motions and a crystal's need to accommodate a given deformation force the crystal lattice (and lattice planes) to reorient as a deformation proceeds. From Equation $5, \boldsymbol{R}_{\boldsymbol{P}}$ is

$$
\boldsymbol{R}_{\boldsymbol{P}}=\boldsymbol{I}+\sum_{\alpha=1}^{\mathrm{SS}} \gamma_{\alpha}\left(\overline{\boldsymbol{s}}_{\boldsymbol{\alpha}} \otimes \overline{\boldsymbol{n}}_{\boldsymbol{\alpha}}\right)_{\mathrm{SKEW}}
$$

During a compression test in this small strain regime, we assume that the total rotation of material (not the crystal lattice) is negligible $(\boldsymbol{R}=\boldsymbol{I})$ since the compression platen constrains rotation of the sample. To illustrate the important relationship between the trajectory $\boldsymbol{v}$ and heterogeneous slip using this model, Figure 4 shows a simple two-dimensional schematic of a single crystal deforming by heterogeneous single slip during a compression experiment. The active slip system's initial slip direction, $\overline{\boldsymbol{s}}_{\boldsymbol{\alpha}}$, slip plane normal, $\overline{\boldsymbol{n}}_{\boldsymbol{\alpha}}$, and their cross product, $\overline{\boldsymbol{m}}_{\boldsymbol{\alpha}}$, are labeled. The crystal has been divided into three volumes that shear by varying amounts, $\gamma_{\alpha}$. Reasons for the differences in shear strain across different crystal volumes will be discussed later, but for now, variation in shear strain is assumed to exist. Material planes initially perpendicular 
to the compression direction, $\boldsymbol{e}_{\boldsymbol{y}}^{S}$, are marked with dashed lines, while the diagonal lines across the volumes mark a set of lattice planes initially normal to $\overline{\boldsymbol{n}}$. In Figure 4A, the deformation is decomposed into configurations of crystal defined by $\boldsymbol{F}_{\boldsymbol{P}}$ and $\boldsymbol{R}^{*}$. In the intermediate configuration, the material volume (but not the lattice) has rotated by $\frac{\gamma_{\alpha}}{2}$. In order for there to be no net rotation of material, each volume must rotate back (with the lattice) by $-\frac{\gamma_{\alpha}}{2}$. Since each volume sheared by varied amounts, a distribution of lattice plane orientation is now present in the crystal.

Figure 4B shows the plane normals in the current (deformed) configuration plotted on a pole figure. The plane normal distribution has spread along $\boldsymbol{v}$ and $\boldsymbol{v}$ is perpendicular to both $\overline{\boldsymbol{s}}_{\boldsymbol{\alpha}}$ and $\overline{\boldsymbol{n}}_{\boldsymbol{\alpha}}$. In the single slip case, $\boldsymbol{t}$ is equal to $\overline{\boldsymbol{m}}_{\boldsymbol{\alpha}}$ and the misorientation angle $\Delta \phi$ is equal to $\frac{\left(\Delta \gamma_{\alpha}\right)}{2}$. The quantity $\Delta \gamma_{\alpha}$ is the maximum difference in shear strain between the most and least sheared crystal volumes. Therefore, during single slip:

$$
\boldsymbol{v}=\frac{\left(\Delta \gamma_{\alpha}\right)}{2} \overline{\boldsymbol{m}}_{\alpha} \times \overline{\boldsymbol{n}}
$$

and $\boldsymbol{w}=\frac{\left(\Delta \gamma_{\alpha}\right)}{2} \boldsymbol{t}_{\boldsymbol{\alpha}}$. The magnitude of the trajectory $\boldsymbol{v}$ is related to the magnitude of heterogeneous shear within the crystal, while the misorientation axis $\boldsymbol{t}$ is related to the active slip system (or slip systems). The value $\Delta \gamma_{\alpha}$ is a measure of the heterogeneity of slip activity on the $\alpha$ slip system and the ultimate goal of this model.

The trajectory $\boldsymbol{v}$ is readily related to activity on multiple slip systems. Using Equation 6 and assuming that $\boldsymbol{R}=\boldsymbol{I}$, we find that

$$
\boldsymbol{R}^{*}=\boldsymbol{I}-\sum_{\alpha=1}^{\mathrm{SS}} \gamma_{\alpha}\left(\overline{\boldsymbol{s}}_{\boldsymbol{\alpha}} \otimes \overline{\boldsymbol{n}}_{\boldsymbol{\alpha}}\right)_{\mathrm{SKEW}}=\boldsymbol{I}+\sum_{\alpha=1}^{\mathrm{SS}} \frac{\gamma_{\alpha}}{2}\left(\boldsymbol{m}_{\boldsymbol{\alpha}} \times \boldsymbol{I}\right)
$$

and through manipulation of Equation 8, $\boldsymbol{v}$ is:

$$
\boldsymbol{v}=\left(\sum_{\alpha=1}^{\mathrm{SS}} \frac{\Delta \gamma_{\alpha}}{2} \overline{\boldsymbol{m}}_{\boldsymbol{\alpha}}\right) \times \overline{\boldsymbol{n}}
$$

when multiple slips systems are active. The misorientation vector $\boldsymbol{w}$ is a linear combination of the maximum differences in shear strain $\Delta \gamma_{\alpha}$ and the directions $\overline{\boldsymbol{m}}_{\boldsymbol{\alpha}}$ associated with each slip system. As would be expected, Equation 9 reduces to Equation 7 if only a single slip system is active. Using measurements of $\boldsymbol{v}$, the magnitudes of slip heterogeneity, $\Delta \gamma_{\alpha}$, on multiple slip 
systems is approximated. Remembering that the vector $\boldsymbol{v}$ approximates an arc on a sphere, once

a trajectory becomes visibly curved on the orientation pole figure, heterogeneous slip can not be decomposed into its constitutive parts, since the description of $\boldsymbol{R}_{\boldsymbol{P}}$ in Equation 8 is not valid.

Critically for interpreting SCPF data, when a trajectory $\boldsymbol{v}$ exists on a SCPF, crystal volumes are plastically deforming with a fixed combination of slip systems by varying amounts. The combination of slip systems produces $\boldsymbol{t}$ while $\Delta \phi$ is produced by variation of the amount of shear strain $\left(\Delta \gamma_{\alpha}\right)$ on those slip systems. If multiple $\boldsymbol{v}_{\boldsymbol{\beta}}$ exist on a SCPF (and therefore multiple $\boldsymbol{t}_{\boldsymbol{\beta}}$ within the volume of interest), then different sub-volumes of crystal are deforming with different combinations of slip systems.

\section{Diffracted Intensity Measurement and Single Crystal Pole Figure Gen- eration}

The measurement of SCPFs builds on previous work measuring pole figures from polycrystals at synchrotron sources using area detectors [31, 32, 33]. This section describes the geometry of the diffraction experiment and the process of producing SCPFs from diffraction images taken on an area detector (outlined in Figure 5). The incoming X-ray beam with wavelength $\lambda$ and wave vector $\boldsymbol{k}_{\boldsymbol{i}}$ (the direction of $\boldsymbol{k}$ is the direction of propagation of the X-ray beam and $\|\boldsymbol{k}\|=\frac{2 \pi}{\lambda}$ ) travels along the $-\boldsymbol{e}_{\boldsymbol{z}}^{L}$ direction, while diffracted X-ray beams, $\boldsymbol{k}_{\boldsymbol{o}}$, intercept the area detector sitting a distance $D$ behind the sample. During the experiment, the sample is rotated about $\boldsymbol{e}_{y}^{L}$. Diffracted intensity, $I$, measured at the detector is parameterized by the angles, $2 \theta, \eta$, and $\omega$. The angle $2 \theta$ at which diffracted intensity is emitted, is determined from the radial position of the diffracted intensity on the detector and the sample to detector distance. The azimuthal angle, $\eta$, is measured counter clockwise from $\boldsymbol{e}_{x}^{D E T}$ on the area detector. The diffractometer angle, $\omega$, is the angle about $\boldsymbol{e}_{\boldsymbol{y}}^{L}$ that the sample was rotated when a diffraction image was taken. At $\omega=0^{\circ}$ the laboratory and sample coordinate systems are in coincidence. The instrument is calibrated using a standard $\mathrm{CeO}_{2}$ calibrant.

For a fixed sample orientation in the laboratory coordinate system, diffraction occurs when the Laue diffraction condition:

$$
\boldsymbol{k}_{\boldsymbol{o}}-\boldsymbol{k}_{\boldsymbol{i}}=\frac{2 \pi}{d} \boldsymbol{n}
$$


is satisfied, where $d$ is the lattice plane spacing. Equation 10 links the direction along which

diffracted intensity is emitted $\hat{\boldsymbol{k}}_{\boldsymbol{o}}$ and the orientation of lattice planes $\boldsymbol{n}$ in a diffracting crystal volume. From Equation 10 and the constraint that the X-ray scattering is elastic, it is found that only lattice planes oriented $90^{\circ}-\theta$ from $\boldsymbol{k}_{\boldsymbol{i}}$ will diffract [32]. To collect diffracted intensity from sets of lattice planes from all illuminated crystal volumes, the crystal is rotated about $\boldsymbol{e}_{\boldsymbol{y}}^{L}$, while the detector collects intensity across angular intervals $\Delta \omega$ (Figure 5A).

Once a diffraction peak's three dimensional distribution of intensity is collected (parameterized by the diffractometer angles $2 \theta, \eta$, and $\omega)$, the intensity is integrated along $2 \theta$. This operation collects intensity from all volumes of crystal whose lattice planes share a common orientation, but varying spacing, to a single point on the pole figure. The integration is performed by radially integrating diffracted intensity on each diffraction image using a finite element based method [34]. A polar grid of three node triangular elements is laid over the diffracted intensity on an image (Figure 5B). The element density of the finite element mesh is chosen to oversample the diffraction image and place approximately 8 elements per pixel area. The intensity values at nodes are evaluated using a bilinear interpolation from the nearest pixel intensity values. Integration is performed by single quadrature point Gauss integration over each element followed by summation of element intensities in the radial $(2 \theta)$ direction creating a distribution of intensity as a function of only $\eta$ and $\omega$ (Figure $5 \mathrm{C}$ ). In the final SCPF generation step, the integrated diffracted intensity is mapped to the SCPF (Figure 5D) which in the given geometry is described with the relation [15]:

$$
\left\{\boldsymbol{n}^{S}\right\}=\left\{\begin{array}{c}
\cos \omega \cos \eta \cos \theta_{0}+\sin \omega \sin \theta_{0} \\
\sin \eta \cos \theta_{0} \\
-\sin \omega \cos \eta \cos \theta_{0}+\cos \omega \sin \theta_{0}
\end{array}\right\}
$$

where $\theta_{0}$ is the Bragg angle of the set of lattice planes in the undeformed state.

\section{Experiment and Results}

An in-situ diffraction experiment was conducted at the A-2 station of the Cornell High Energy Synchrotron Source (CHESS) to measure single crystal orientation pole figures from a silicon single crystal as it plastically deformed. The silicon crystal was deformed in uniaxial compres- 
sion, and the evolution of the pole figures was tracked with increasing load magnitude. Plastic

deformation was initiated by applying a uniaxial macroscopic compressive stress $\sigma_{\mathrm{MACRO}}$ along $\boldsymbol{e}_{\boldsymbol{y}}^{S}$ at $800^{\circ} \mathrm{C}$ since silicon becomes increasingly ductile past $700^{\circ} \mathrm{C}$ [35]. The sample was cut from a float-zone grown silicon boule such that the [123] crystallographic direction was aligned with $\boldsymbol{e}_{y}^{S}$. With this orientation, the crystal was expected to deform primarily with a single slip system. After etching, the sample size was $0.98 \mathrm{~mm} \times 2.35 \mathrm{~mm} \times 0.98 \mathrm{~mm}$ along $\boldsymbol{e}_{\boldsymbol{x}}^{S}, \boldsymbol{e}_{\boldsymbol{y}}^{S}$, and $\boldsymbol{e}_{z}^{S}$ respectively. The crystal was illuminated by a monochromatic $50.239 \mathrm{keV}$ X-ray beam with a beam size of $1.50 \mathrm{~mm}$ (along $\boldsymbol{e}_{\boldsymbol{x}}^{L}$ ) $\times 0.25 \mathrm{~mm}$ (along $\boldsymbol{e}_{\boldsymbol{y}}^{L}$ ) which was sufficiently large to always illuminate the entire cross section of the sample. The detector used was a GE41RT+ amorphous silicon area detector with $2048 \times 2048$ pixels and $200 \mu \mathrm{m} \times 200 \mu \mathrm{m}$ pixel size. The sample to detector distance $D$ was $1270 \mathrm{~mm}$.

The test was composed of heating the sample to $800^{\circ} \mathrm{C}$ and then measuring $19 \mathrm{SCPFs}$ at each load step from the $\{111\},\{220\}$, and $\{311\}$ families of lattice planes across the available diffractometer rotation range $\left(\omega=\left[-60^{\circ}, 60^{\circ}\right]\right)$. Integrated diffraction images were taken every $0.125^{\circ}$ (defining an experimental uncertainty of measured misorientation of $\pm 0.0625^{\circ}$ ) as the sample rotated. The angular resolution of the area detector used ranged from $0.11^{\circ}$ to $0.06^{\circ}$ for $\{111\}$ and $\{311\}$ pole figures. A small compressive preload ( $-4 \mathrm{MPa}$ ) was applied to the sample prior to heating. The sample was heated using a resistance wire furnace with the temperature maintained by PID control [36]. The load was applied by an electromagnetic actuator mounted on a custom diffractometer. Diffraction data were measured at uniaxial macroscopic stresses $\left(\sigma_{\mathrm{MACRO}}\right)$ of $-10.6,-21.3,-31.9,-42.6$, and $-53.2 \mathrm{MPa}$. The stress-strain data from the compression experiment are shown in Figure 6. At each load step, the applied stress was reduced to $80 \%$ of the maximum stress magnitude and then held as the diffraction data were collected. The magnitude of the applied stress was reduced as the diffraction measurements were made in order to move the stress state off of the single crystal yield surface and reduce creep. However, as can be seen, some material evolution still occurred during diffraction data collection.

Figure 7 shows the evolution of $P_{\overline{2} 02}, P_{\overline{1} \overline{3} 1}$, and $P_{11 \overline{3}}$ (examples of the SCPFs measured) as the deformation proceeded. The SCPFs are from the preload step and at $\sigma_{\mathrm{MACRO}}=-31.9,-42.6$, and $-53.2 \mathrm{MPa}$. The SCPFs from $\sigma_{\mathrm{MACRO}}=-10.6$ and $-21.3 \mathrm{MPa}$ are not shown because there was no measurable pole figure evolution. In the SCPFs shown, the logarithm of each pole density value is shown and normalized so that the maximum value of each SCPF is 1 . The data 
are presented in this manner in order to increase the visibility of features on the SPCFs (notably the full extent of the trajectories $\boldsymbol{v}$ ). The initial positions of the lattice plane normals $\overline{\boldsymbol{n}}$ with respect to the sample coordinate system are shown at the bottom left of the figure. While only three SCPFs are shown, the distributions of lattice plane orientation from other sets of lattice planes exhibited similar behavior.

Two distinct $\boldsymbol{v}$, that develop and extend as the deformation proceeds, are observed on the SCPFs in Figure 7. The expansion of non-zero regions of the SCPFs with increasing load magnitude indicates an increase in the misorientation present within the crystal (since $\boldsymbol{v}$ is proportional to $\Delta \phi$, Equation 1). The two distinct streaking directions on the SCPFs indicate that sub-volumes in the diffraction volume are developing misorientation about two different axes and also deforming using two different combinations of slip systems. The two trajectories are both apparent at the $-31.9 \mathrm{MPa}$ load steps, indicating the two sub-volumes with different misorientation axes develop simultaneously rather than sequentially. The larger trajectory on each SCPF will be referred to as $\boldsymbol{v}_{\mathbf{1}}$, while the shorter will be referred to as $\boldsymbol{v}_{\mathbf{2}}$, and the corresponding misorientation vectors will be referred to as $\boldsymbol{w}_{1}$ and $\boldsymbol{w}_{2}$ respectively.

Using the $\boldsymbol{v}_{\boldsymbol{\beta}}$ from all 19 SCPFs, the misorientation vectors $\boldsymbol{w}_{\boldsymbol{\beta}}$ (comprised of a misorientation angle $\Delta \phi_{\beta}$ and axis $\boldsymbol{t}_{\beta}$ ) were found using a least squares minimization. A detailed description of the minimized function can be found in Appendix B. Figure 8A shows the evolution of $\left|\Delta \phi_{1}\right|$ and $\left|\Delta \phi_{2}\right|$. In the Figure, it is seen that both $\left|\Delta \phi_{1}\right|$ and $\left|\Delta \phi_{2}\right|$ are increasing non-linearly with increasing load magnitude. Figure 8B shows the evolution of $\boldsymbol{t}_{\mathbf{1}}$ and $\boldsymbol{t}_{\mathbf{2}}$ during the experiment. There is minimal evolution of the orientation of $\boldsymbol{t}_{\mathbf{1}}$ and $\boldsymbol{t}_{\mathbf{2}}$ throughout the experiment.

\subsection{Quantifying Heterogeneous Slip Activity}

The experimental results in $\S 5$ showed the development of two distinct trajectories, $\boldsymbol{v}_{\mathbf{1}}$ and $\boldsymbol{v}_{\mathbf{2}}$, on the SCPFs as the silicon single crystal deformed. The existence of these two trajectories indicates that two volumes within the crystal developed misorientation about two axes due to slip activity on two different combinations of slip systems. Now, using the slip kinematics described in $\S 3$, the heterogeneous slip that produced these two misorientation axes is determined. In the analysis, estimates of the resolved shear stress on different slip systems are used to reduce the number of possible active slip systems to three. The heterogeneous slip on these three slip systems is then 
determined.

The primary slip systems of silicon at $800^{\circ} \mathrm{C}$ are of the $<110>(111)$ type [37]. During uniaxial loading, the average resolved shear stress on each slip system, $\tau_{\alpha}$, is approximated using the Schmid factor, $\mathrm{SF}_{\alpha}$, of each slip system. The approximate resolved shear stress on each slip system is found by multiplying the Schmid factor by the uniaxial stress $\left(\tau_{\alpha}=\mathrm{SF}_{\alpha} \sigma_{\mathrm{MACRO}}\right)$ with the Schmid factor given by:

$$
\mathrm{SF}_{\alpha}=\left|\bar{s}_{\alpha y}^{S} \bar{n}_{\alpha y}^{S}\right|
$$

Table 1 lists the twelve primary slip systems and their corresponding Schmid factors when the crystal is oriented with the [123] direction along the loading axis. The slip systems are named according to convention defined in $[38,39]$ where letters correspond to slip planes and numbers to slip directions. The deformation of the silicon crystal is assumed to be mostly accommodated by activity on the primary slip system $\mathrm{B} 4\left(\mathrm{SF}_{\alpha}=0.47\right)$, with additional slip activity on slip systems B5 and $\mathrm{A} 3\left(\mathrm{SF}_{\alpha}=0.35\right)$ because these three slip systems have the largest Schmid Factors. This assumption is discussed further in $§ 6.2$. Therefore, the misorientation vectors $\boldsymbol{w}_{\boldsymbol{\beta}}$ are assumed to be a linear combination of the axes $\overline{\boldsymbol{m}}_{\boldsymbol{\alpha}}$ and spread of shear strain $\Delta \gamma_{\alpha}$ associated with B4, B5, and A3 slip systems.

Using Equations 1 and 9, $\Delta \gamma_{\alpha}$ are determined from the values of $\boldsymbol{w}$ found in $\S 5$. With three slip systems, $\Delta \gamma_{\alpha}$ can be uniquely calculated. The evolution of $\Delta \gamma_{\alpha}$ associated with $\boldsymbol{w}_{1}$ and $\boldsymbol{w}_{2}$ are displayed in Figure 9. As indicated by the large $\Delta \gamma_{B 4}$ magnitudes, most of the misorientation is found to be due to heterogeneity of slip on the primary slip system B4 for both $\boldsymbol{w}_{1}$ and $\boldsymbol{w}_{2}$. This slip behavior was expected for the crystal orientation tested. The heterogeneity of shear on slip system B4 (filled and open triangles) also appears to be increasing exponentially with applied load. An appreciable $\Delta \gamma_{B 5}$ value (open circles) is observed in $\boldsymbol{w}_{2}$ when compared to $\Delta \gamma_{A 3}$. The relative magnitudes of $\Delta \gamma_{B 5}$ and $\Delta \gamma_{A 3}$ indicate that there is more activity on slip system B5 than A3, since $\Delta \gamma_{A 3}$ is small in both $\boldsymbol{w}_{1}$ and $\boldsymbol{w}_{2}$.

From Figure 9, the combinations of active slip systems in the sub-volumes associated with the two $\boldsymbol{v}_{\boldsymbol{\beta}}$ on the SCPFs were determined. First, the volume of crystal associated with the larger trajectory $\left(\boldsymbol{v}_{\mathbf{1}}\right)$ on the SCPFs is deforming using the B4 slip system only. The second sub-volume associated with the shorter trajectory $\left(\boldsymbol{v}_{\mathbf{2}}\right)$ on each SCPF is deforming using a combination of 
the B4 and B5 slip systems. The additional activity of B5 in the second sub-volume produces the difference in trajectory directions between $\boldsymbol{v}_{\mathbf{2}}$ and $\boldsymbol{v}_{\mathbf{1}}$ observed in the pole figure data.

\section{Discussion}

True prediction and modeling of slip driven failure will require require major advances in our ability to measure heterogeneously slip processes. To begin to meet this need, a new experimental method was presented in this paper for quantifying heterogeneous slip in individual deforming crystals. The method aimed to address some of the major issues surrounding our ability to experimentally measure slip: the need to make time resolved measurements and the need to probe within the bulk of deforming samples. The kinematic model presented gives a direct method to interpret raw diffraction data in terms of shear strains relevant to the mechanical response using a multiplicative decomposition of a crystal's deformation. The method took advantage of the large amount of lattice plane orientation data rapidly acquired at a synchrotron source to understand the evolution of the mechanical material response.

The heterogeneity of shear strains in two sub-volumes of a single crystal that were deforming using different combinations of active slip systems were quantified simultaneously from the single crystal pole figures. The ability to independently study sub-volumes of a crystal was a natural benefit of analyzing distributions of lattice orientation to infer slip activity: the different combinations of active slip systems caused the distribution of orientation on the SCPFs to divide into two distinct regions of pole density. Probing the entire sample cross section ensured that slip activity throughout the sample was accounted for (not just at the surface).

The relatively short data collection times per load step (10-30 minutes), made in-situ diffraction measurements during deformation possible. Highlighting understanding of slip that could only be obseerved with time resolved measurements, we were able to observe that the two subvolumes deforming with different combinations of slip systems formed simultaneously rather than the sequentially because measurements were made at multiple load steps.

To develop the method in this work, a single crystal was deformed, but extending this method to studying the in-situ evolution of sub-crystal scale slip in many grains is a promising avenue of further research. A strength of this method is that in-situ slip analysis can be performed on an instrument already often used for the study of lattice orientation and strain in individual 
grains in polycrystalline samples. Also, with improvements to X-ray source brightness and detector efficiency, reduction of measurement times making the generation of 'movies' of slip processes feasible. One could even foresee being able to continuously monitor slip activity in deeply embedded grains in polycrystals.

\subsection{Extending the Use of the Slip Analysis}

The kinematic analysis presented in this paper was consistent with a single crystal in compression and enabled the quantification of heterogeneous slip activity from SCPF data. Ideally, a similar analysis could be applied to the study of individual grains in polycrystals that are not expected to have uniaxial stress states. In the single crystal compression analysis, we assumed that the rotation of the material was negligible $(\boldsymbol{R}=\boldsymbol{I})$, but the crystal lattice was still able to rotate. This important assumption enabled the association of pole figure evolution directly with heterogeneous slip activity. This kinematic analysis of deformation is analogous to a micro/macro linking hypothesis, since we assume that the rotation of each volume element of material is equivalent to a macroscopic response. If we were to analyze SCPFs from a grain embedded within a polycrystal, we could preliminarily assume that the rotation (or spin) of each grain is equivalent to the macroscopic value (applying the extended Taylor hypothesis). Making this assumption would enable the determination of $\Delta \gamma_{\alpha}$ from a deforming grain within a polycrystal. However, we reiterate that $\boldsymbol{w}$ can be measured during any deformation, and assumptions made about the deformation of a crystal are only required to estimate slip activity $\left(\Delta \gamma_{\alpha}\right)$ from the misorientation vector $\boldsymbol{w}$.

Another important issue to address is the choice of $\overline{\boldsymbol{m}}_{\boldsymbol{\alpha}}$ used to construct the misorientation vector $\boldsymbol{w}$. In $\S 3$, the vectors $\boldsymbol{w}$ describing the misorientation about an axis were described as linear combinations of directions $\overline{\boldsymbol{m}}_{\boldsymbol{\alpha}}$. Any combination of three $\overline{\boldsymbol{m}}_{\boldsymbol{\alpha}}$ that are not co-planar (an example of three coplanar systems are B2, B4 and B5) can be used to generate a general $\boldsymbol{w}$. An equivalent statement is that only three independent slip systems are necessary to produce a general $\boldsymbol{R}_{\boldsymbol{P}}$ in the model employed. Any more than three slip systems used to generate $\boldsymbol{w}$ will produce non-unique solutions for values of $\Delta \gamma_{\alpha}$. To address this problem, the choice of $\overline{\boldsymbol{m}}_{\boldsymbol{\alpha}}$ must be informed with knowledge of the average crystal stress state in the crystal (readily found using X-ray diffraction during the same experiment) so that aphysical slip activity is not 
found. This should not pose a problem in the early stages of plastic deformation, when most

loading conditions will produce variation in the resolved shear stresses on different slip systems. In addition, transmission electron microscopy observations of sub-crystal scale slip behavior indicate that sub-volumes of crystal rarely deform with more than three active slip systems [40]. Although we note that if the stress state of a crystal volume is already in a vertex of the single crystal yield surface (where five slip systems are simultaneously active), the methodology presented cannot uniquely determine slip activity on different slip systems.

\subsection{Causes of Intracrystal Heterogeneous Slip Activity}

For any heterogeneous plastic deformation to occur, there must be heterogeneity of stress state throughout the crystal. Typically within a grain in a polycrystal, the complex loading at the grain's boundary produces heterogeneity of stress throughout the grain. In the case of the single crystal compression experiment described in this paper, the interface between the sample and platen has long been known to cause departures from uniaxial stress near the platen [41]. Also, compression tests of brick-shaped single crystals with different ratios of cross sectional area to height have been shown to have different stress-strain responses, indicating that sample shape also affects the heterogeneity of stress $[6,42]$. Both of these effects are assumed to produce the deviations from uniaxial stress near the center of the sample where diffraction measurements were made. However, the deviations from uniaxial stress are not sufficient to change the primary slip system from B4 (as predicted by the Schmid Factor analysis) to another slip system.

In addition to variation of stress state, the constitutive response of the material studied will also affect the development of misorientation within a crystal. A phenomenological power law model is often used to describe the constitutive relationship relating the applied shear stress, $\tau_{\alpha}$, and the shearing rate of each slip system, $\dot{\gamma}_{\alpha}$, in plastically deforming crystals [43]

$$
\dot{\gamma}_{\alpha}=\dot{\gamma}_{0}\left(\frac{\tau_{\alpha}}{g_{\alpha}}\right)\left|\frac{\tau_{\alpha}}{g_{\alpha}}\right|^{(1 / m-1)}
$$

where $g_{\alpha}$ is the slip system strength, $m$ is the power law exponent, and $\dot{\gamma}_{0}$ is a fixed strain rate scaling coefficient. Typically when modeling the plastic deformation of crystalline materials, the power law exponent $m$ ranges from between 0.01 to 0.25 . There exist two important consequences of the power law relationship between resolved shear stress and shearing rate. First, is that unless 
the resolved shear stresses on different slip systems are very close in magnitude, their respective shearing rates (and total shear) will be significantly different. Therefore for most stress states, there is a rapid decay of shearing rate of the most active to least active slip systems. This rapid decay of slip activity with decreasing resolved shear stress is why slip is only attributed to the B4, B5, and A3 slip systems in the analysis in this paper. The second consequence is that small differences in resolved shear stress on a single slip system across a crystal will result in very large differences in shearing rates. Thus, large distributions of lattice orientation can be generated by relatively small differences in $\tau_{\alpha}$ and the variation of activity of slip systems across a crystal due to stress heterogeneity drives the misorientation evolution observed in the experiment.

The strain rate scaling coefficient, $\dot{\gamma}_{0}$, in the constitutive relation described in Equation 13 has been linked to the mobile dislocation density of a slip system $[44,45]$. When modeling a metallic system, $\dot{\gamma}_{0}$, can be modeled as a constant through the deformation, equivalent to assuming the mobile density is at steady state at the beginning of the test. However, for the silicon tested (which had a dislocation density several orders of magnitude lower than a metal at the beginning of the test), the mobile density must have rapidly evolved during testing. The evolution of the mobile dislocation density in the silicon may have also influenced the slip activity observed in the experiment. The heterogeneous slip activity $\left(\Delta \gamma_{\alpha}\right)$ determined from the experimental data in this work only relied on kinematics and some knowledge of the average crystal stress state. An important utility of the analysis in this work is that different hypotheses about the material constitutive response can be tested using the experimental data.

\section{Summary}

In this paper, single crystal orientations pole figures (SCPFs) were used to quantify heterogeneity of crystallographic slip. Heterogeneity of slip activity within the crystal caused a distribution of lattice orientation to develop, which manifested as distinct streaking observed on the measured orientation pole figures. A multiplicative decomposition of the crystal kinematics provided a link between magnitudes of heterogeneous plastic deformation and single crystal pole figure evolution that enabled in-situ quantitative analysis of the evolution of the slip activity throughout the bulk of the crystal. In the experiment, the streaking on the SCPFs was found to related to different combinations of slip systems being active in two sub-volumes of crystal. In one sub-volumes only 
the B4 slip system was active, and in the second sub-volume. heterogeneous activity on two slip

systems, B4 and B5 was measured. Evidence of appreciable heterogeneous activity on a third slip system, A3, which would be expected from a simple Schmid factor analysis, was not found to be present. The results of this work provide confidence that this methodology is capable of identifying heterogeneous slip activity on multiple slip systems within deforming crystals.

\section{Acknowledgements}

The experiment was conducted at the Cornell High Energy Synchrotron Source (CHESS), which is supported by the National Science Foundation and the National Institutes of Health/National Institute of General Medical Sciences under NSF Award No. DMR-1332208. Darren Pagan was supported by a GRA position at CHESS. This work was performed partially under the auspices of the U.S. Department of Energy by Lawrence Livermore National Laboratory under Contract DE-AC52-07NA27344 (LLNL-JRNL-679683). The authors wish to thank Professors Paul Dawson and Armand Beaudoin for many helpful discussions and guidance, Mark Obstalecki for help performing the silicon compression experiment, and Dr. Jacob Ruff for his support as staff scientist at the A-2 station at CHESS.

\section{References}

[1] T.R. Bieler and S.L Semiatin. The origins of heterogeneous deformation during primary hot working of ti-6al-4v. International Journal of Plasticity, 18(9):1165 - 1189, 2002.

[2] L. Wang, Y. Yang, P. Eisenlohr, T.R. Bieler, M.A. Crimp, and D.E. Mason. Twin nucleation by slip transfer across grain boundaries in commercial purity titanium. Metallurgical and Materials Transactions A, 41(2):421-430, 2010.

[3] P. B. Hirsch, R. W. Horne, and M. J. Whelan. Lxviii. direct observations of the arrangement and motion of dislocations in aluminium. Philosophical Magazine, 1(7):677-684, 1956.

[4] E. M. Schulson. Electron channelling patterns in scanning electron microscopy. Journal of Materials Science, 12(6):1071-1087, 1977. 
[5] A.F. Joffe and M.V. Kirpitcheva. Xviii. röntgenograms of strained crystals. Philosophical

[6] Y.Nakada U.F. Kocks and B.Ramaswami. Compression testing of fcc crystals. Transactions of the Metallurgical Society of AIME, 230:1005-1009, 1964.

[7] J.F. Nye. Some geometric relations in dislocated crystals. Acta Metallurgica, 1:153-162, 1953.

[8] T. Ungar, O. Castelnau, G. Ribárik, M. Drakopoulos, J.L. Béchade, T. Chauveau, A. Snigirev, I. Snigireva, C. Schroer, and B. Bacroix. Grain to grain slip activity in plastically deformed zr determined by x-ray micro-diffraction line profile analysis. Acta Materialia, $55(3): 1117-1127,2007$.

[9] T. Ungar, M.G. Glavicic, L. Balogh, K. Nyilas, A.A. Salem, G. Ribárik, and S.L. Semiatin. The use of x-ray diffraction to determine slip and twinning activity in commercial-purity (cp) titanium. Materials Science and Engineering: A, 493(1-2):79 - 85, 2008. Mechanical Behavior of Nanostructured Materials, a Symposium Held in Honor of Carl Koch at the TMS Annual Meeting 2007, Orlando, Florida.

[10] T. Ungar, G. Ribárik, G. Zilahi, R. Mulay, U. Lienert, L. Balogh, and S. Agnew. Slip systems and dislocation densities in individual grains of polycrystalline aggregates of plastically deformed coti and cozr alloys. Acta Materialia, 71(0):264 - 282, 2014.

[11] G.E. Ice and R.I. Barabash. Chapter 79 white beam microdiffraction and dislocations gradients. volume 4 of Dislocations in Solids, pages 261-362. Elsevier, 2007.

[12] D.C. Pagan and M.P. Miller. Connecting heterogeneous single slip to diffraction peak evolution in high-energy monochromatic X-ray experiments. Journal of Applied Crystallography, 47(3):887-898, 2014.

[13] L. Margulies, T. Lorentzen, H.F. Poulsen, and T. Leffers. Strain tensor development in a single grain in the bulk of a polycrystal under loading. Acta Materialia, 50(7):1771 - 1779, 2002 . 
[14] U. Lienert, M.C. Brandes, J.V. Bernier, J. Weiss, S.D. Shastri, M.J. Mills, and M.P. Miller. In situ single-grain peak profile measurements on ti-7al during tensile deformation. Materials Science and Engineering: A, 524(1-2):46 - 54, 2009.

[15] J.V. Bernier, N.R. Barton, U. Lienert, and M.P. Miller. Far-field high-energy diffraction microscopy: a tool for intergranular orientation and strain analysis. The Journal of Strain Analysis for Engineering Design, 46(7):527-547, 2011.

[16] R. M. Suter, D. Hennessy, C. Xiao, and U. Lienert. Forward modeling method for microstructure reconstruction using x-ray diffraction microscopy: Single-crystal verification. Review of Scientific Instruments, 77(12):123905, 2006.

[17] G. Johnson, A. King, M.G. Honnicke, J. Marrow, and W. Ludwig. X-ray diffraction contrast tomography: a novel technique for three-dimensional grain mapping of polycrystals. II. The combined case. Journal of Applied Crystallography, 41(2):310 - 318, 2008.

[18] B. Jakobsen, H.F. Poulsen, U. Lienert, J. Almer, S.D. Shastri, H.O. Sørensen, C. Gundlach, and W. Pantleon. Formation and subdivision of deformation structures during plastic deformation. Science, 312(5775):889-892, 2006.

[19] H.F. Poulsen. Three-Dimension X-Ray Diffraction Microscopy. Springer, 1st edition, 2004.

[20] M.P. Miller and P.R. Dawson. Understanding local deformation in metallic polycrystals using high energy x-rays and finite elements. Current Opinion in Solid State and Materials Science, (0), 2014.

[21] H.R. Wenk. Measurement of pole figures. In H.R. Wenk, editor, Preferred Orientation in Deformed Metals and Rocks: An Introduction to Modern Texture Analysis, pages 73-103. Academic Press, 1986.

[22] H. Behnken. Strain-Function Method for the Direct Evaluation of Intergranular Strains and Stresses. physica status solidi (a), 177(2):401-418, 2000.

[23] Y.D. Wang. Stress-Orientation Distribution Function (SODF) - Description and Symmetry. In A. J. Beottger, R. Delhez, and E. J. Mittemeijer, editors, ECRS 5 : proceedings of the Fifth European Conference on Residual Stresses, pages 66-71, Delft-Noordwijkerhout, The Netherlands, 2000. 
[24] M.P. Miller, J. V. Bernier, J.S. Park, and A. Kazimirov. Experimental measurement of lattice strain pole figures using synchrotron x rays. Review of Scientific Instruments, 76(11):113903, 2005.

[25] H. J. Bunge. Texture Analysis in Materials Science. Butterworths, London, 1982.

[26] N.R. Barton and J.V. Bernier. A method for intragranular orientation and lattice strain distribution determination. Journal of Applied Crystallography, 45(6):1145-1155, 2012.

[27] P. Hansen, H. Sørensen, Z. Sükösd, and H.F. Poulsen. Reconstruction of single-grain orientation distribution functions for crystalline materials. SIAM Journal on Imaging Sciences, 2(2):593-613, 2009.

[28] R.J. Asaro. Crystal plasticity. Journal of Applied Mechanics, 50(4b):921-934, 1983.

[29] D.J. Bammann and G.C. Johnson. On the kinematics of finite-deformation plasticity. Acta Mechanica, 70(1-4):1-13, 1987.

[30] U.F. Kocks and H. Chandra. Slip geometry in partially constrained deformation. Acta Metallurgica, 30(3):695-709, 1982.

[31] L. Wcislak, H. Klein, H. J. Bunge, U. Garbe, T. Tschentscher, and J. R. Schneider. Texture analysis with high-energy synchrotron radiation. Journal of Applied Crystallography, 35(1):82-95, 2002.

[32] H.-R. Wenk and S. Grigull. Synchrotron texture analysis with area detectors. Journal of Applied Crystallography, 36(4):1040-1049, 2003.

[33] J.V. Bernier, M.P. Miller, and D.E. Boyce. A novel optimization-based pole-figure inversion method: comparison with WIMV and maximum entropy methods. Journal of Applied Crystallography, 39(5):697-713, Oct 2006.

[34] J.C. Schuren and M.P. Miller. Quantifying the uncertainty of synchrotron-based lattice strain measurements. The Journal of Strain Analysis for Engineering Design, 46(7):663$681,2011$. 
[35] H. Siethoff. Chapter 3.6 macroscopic mechanical behaviour of si at high temperature. Properties of Crystalline Silicon, pages 122-135. Institution of Engineering and Technology, 1999.

[36] B.B. Oswald, J.C. Schuren, D.C. Pagan, and M.P. Miller. An experimental system for high temperature x-ray diffraction studies with in situ mechanical loading. Review Of Scientific Instruments, 84(3), 2013.

[37] H. Alexander and P. Haasen. Dislocations and plastic flow in the diamond structure. volume 22 of Solid State Physics, pages 27 - 158. Academic Press, 1969.

[38] E. Schmid and W. Boas. Plasticity of Crystals. F.A. Hughes and Co., 1950.

[39] S.K. Basinski and Z.S. Basinski. Chapter 16 plastic deformation and work hardening. volume 13 of Dislocations in Solids, pages 499 - 601. Elsevier, 1979.

[40] D. Kuhlmann-Wilsdorf, J. T. Moore, E. A. Starke, and S. S. Kulkarni. Deformation bands, the leds theory, and their importance in texture development: Part i. previous evidence and new observations. Metallurgical and Materials Transactions A, 30(9):2491-2501, 1999.

[41] G.I. Taylor. The distortion of crystal of aluminum under compression. part ii. distortion by double slipping and changes in orientation of crystal axes during compression. Proceedings of the Royal Society A, 116(773):16-38, 1927.

[42] U.F. Kocks and T.J. Brown. Latent hardening in aluminum. Acta Metallurgica, 14(2):87-98, 1966.

[43] J.W. Hutchinson. Bounds and self-consistent estimates for creep of polycrystalline materials. Proceedings of the Royal Society of London, 348(1652):101-127, 1976.

[44] L.P. Kubin, K. Chihab, and Y. Estrin. The rate dependence of the portevin-le chatelier effect. Acta Metallurgica, 36(10):2707 - 2718, 1988.

[45] K.J.H Al-Fadhalah, C.-M. Li, A.J. Beaudoin, D.A. Korzekwa, and I.M. Robertson. Microplastic processes developed in pure ag with mesoscale annealing twins. Acta Materialia, 56(19):5764 - 5774, 2008. 


\section{A Derivation of Equation 1}

This section derives Equation 1, which relates the spread of a distribution of plane normals on a single crystal orientation pole figure (SCPF) $\boldsymbol{v}$, to the axis $\boldsymbol{t}$ about which a set of crystal volumes is developing misorientation, $\Delta \phi$ the angle of misorientation of the crystallites, and $\overline{\boldsymbol{n}}$ the lattice plane normal orientation of the crystallites in the reference configuration. A diagram of the quantities used to describe $\boldsymbol{v}$ are found in Figure 10. The vector $\boldsymbol{v}$ is defined as the difference between the plane normal positions at the start $\left(\boldsymbol{n}_{\boldsymbol{S}}\right)$ and end $\left(\boldsymbol{n}_{\boldsymbol{E}}\right)$ of the trajectory $\boldsymbol{v}$.

$$
v=n_{E}-n_{S}
$$

The rotation $\boldsymbol{R}_{M}$ rotates $\boldsymbol{n}_{S}$ by $\Delta \phi$ about $\boldsymbol{t}$ (see Figure 10). If the crystal volumes contributing to the SCPF are misoriented about $\boldsymbol{t}$ by a small angle $\Delta \phi$, it is found that:

$$
\begin{gathered}
n_{E}=\boldsymbol{R}_{M}(\Delta \phi, \boldsymbol{t}) \cdot \boldsymbol{n}_{S} \\
\boldsymbol{n}_{\boldsymbol{E}} \approx(\boldsymbol{I}+(\Delta \phi \boldsymbol{t} \times \boldsymbol{I})) \cdot \boldsymbol{n}_{S} \\
n_{E} \approx \Delta \phi \boldsymbol{t} \times \boldsymbol{n}_{S}+\boldsymbol{n}_{S}
\end{gathered}
$$

A primary interest is the evolution of a set of crystallites having a single orientation to having a distribution of orientations as it deforms. The plane normals of each volume of crystal rotate from an initial lattice plane orientation $\overline{\boldsymbol{n}}$ to their current orientation $\boldsymbol{n}$. The relationship between the two plane normal orientations is given by:

$$
n=R^{*} \cdot \bar{n}
$$

where $\boldsymbol{R}^{*}$ is the rotation of the crystal lattice. The lattice rotation $\boldsymbol{R}^{*}$ of each crystal volume is different, and the variation of $\boldsymbol{R}^{*}$ produces the distribution of plane normals observed on the SCPF. The trajectory $\boldsymbol{v}$ can also be described in terms of $\boldsymbol{R}^{*}$ and the initial plane normal orientation $\overline{\boldsymbol{n}}$ (specifically denoting the rotations $\boldsymbol{R}_{\boldsymbol{E}}^{*}$ and $\boldsymbol{R}_{S}^{*}$ which rotates $\overline{\boldsymbol{n}}$ to $\boldsymbol{n}_{\boldsymbol{E}}$ and $\boldsymbol{n}_{S}$ respectively):

$$
v=n_{E}-n_{S}=R_{E}^{*} \cdot \bar{n}-R_{S}^{*} \cdot \bar{n} .
$$




$$
\boldsymbol{v}=\left(\boldsymbol{R}_{M}(\Delta \phi, \boldsymbol{t}) \cdot \boldsymbol{R}_{S}^{*}-\boldsymbol{R}_{S}^{*}\right) \cdot \overline{\boldsymbol{n}}
$$

Now, if both $\boldsymbol{R}_{\boldsymbol{M}}$ and $\boldsymbol{R}_{\boldsymbol{S}}^{*}$ are small rotations $\left(\boldsymbol{R}_{\boldsymbol{M}} \approx \boldsymbol{I}+(\phi \boldsymbol{t} \times \boldsymbol{I})\right.$ and $\boldsymbol{R}_{\boldsymbol{S}}^{*} \approx \boldsymbol{I}+\boldsymbol{r}_{\boldsymbol{S}}^{*}$, where $\boldsymbol{r}_{\boldsymbol{S}}^{*}$ is a small skew symmetric tensor) then

$$
\boldsymbol{R}_{M}(\Delta \phi, \boldsymbol{t}) \cdot \boldsymbol{R}_{S}^{*}-\underline{\boldsymbol{R}}_{S}^{*} \approx(\boldsymbol{I}+(\phi \boldsymbol{t} \times \boldsymbol{I})) \cdot\left(\boldsymbol{I}+\boldsymbol{r}_{\boldsymbol{S}}^{*}\right)-\left(\boldsymbol{I}+\boldsymbol{r}_{\boldsymbol{S}}^{*}\right)
$$

After neglecting the second order terms it can be found that:

$$
\underline{\boldsymbol{R}}_{M}(\Delta \phi, \boldsymbol{t}) \cdot \underline{\boldsymbol{R}}_{S}^{*}-\underline{\boldsymbol{R}}_{S}^{*} \approx(\phi \boldsymbol{t} \times \boldsymbol{I})
$$

Inserting this result back into Equation 18, $\boldsymbol{v}$ is found to be:

$$
\boldsymbol{v}=(\phi \boldsymbol{t} \times \boldsymbol{I}) \cdot \overline{\boldsymbol{n}}
$$

\section{B Description of Least Squares Minimization to Determine Misorienta- tion from Single Crystal Pole Figures}

The misorientation vectors $\boldsymbol{w}_{\boldsymbol{\beta}}$ which best fit the trajectories $\boldsymbol{v}_{\boldsymbol{\beta}}$ measured on single crystal orientation pole figures are found using a least squares minimization. In the minimization, two quantities are minimized simultaneously in order for the minimization to be robust. The quantities to be described are illustrated in Figure 11. The first quantity is a scalar distance $u_{i}$ defined as:

$$
u_{i}=\left\|\boldsymbol{v}_{\boldsymbol{\beta} i}-\boldsymbol{w}_{\boldsymbol{\beta}} \times \overline{\boldsymbol{n}}_{\boldsymbol{i}}\right\|
$$

where $\boldsymbol{v}_{\boldsymbol{\beta} \boldsymbol{i}}$ and $\overline{\boldsymbol{n}}_{\boldsymbol{i}}$ are $\boldsymbol{v}_{\boldsymbol{\beta}}$ and $\overline{\boldsymbol{n}}$ of the $i$ th pole figure. The quantities $\boldsymbol{v}_{\boldsymbol{\beta} \boldsymbol{i}}$ and $\overline{\boldsymbol{n}}_{\boldsymbol{i}}$ are both measured quantities, while $\boldsymbol{w}_{\boldsymbol{\beta}}$ is the quantity to be optimized. The second quantity is the angle $\zeta$ defined as:

$$
\zeta_{\beta i}=\arccos \left(\frac{\boldsymbol{v}_{\boldsymbol{\beta} \boldsymbol{i}} \cdot\left(\boldsymbol{w}_{\boldsymbol{\beta}} \times \overline{\boldsymbol{n}}_{\boldsymbol{i}}\right)}{\left\|\boldsymbol{v}_{\boldsymbol{\beta} \boldsymbol{i}}\right\|\left\|\boldsymbol{w}_{\boldsymbol{\beta}} \times \overline{\boldsymbol{n}}_{\boldsymbol{i}}\right\|}\right)
$$

The objective function, $f\left(\boldsymbol{w}_{\boldsymbol{\beta}}\right)$, minimized is then

$$
f\left(\boldsymbol{w}_{\boldsymbol{\beta}}\right)=\sum_{i=1}^{h k l}\left(u_{\beta i}^{2}+\zeta_{\beta i}^{2}\right)
$$


The first term in the function minimized is the distance between the measured and optimized

1 2 $\boldsymbol{v}$, while the second term is the angle between these two vectors. The minimization of both $u_{i}$ and $\zeta_{i}$ terms (as opposed to $u_{i}$ only) improves the robustness of the function minimization by reducing the influence of experimental noise. 


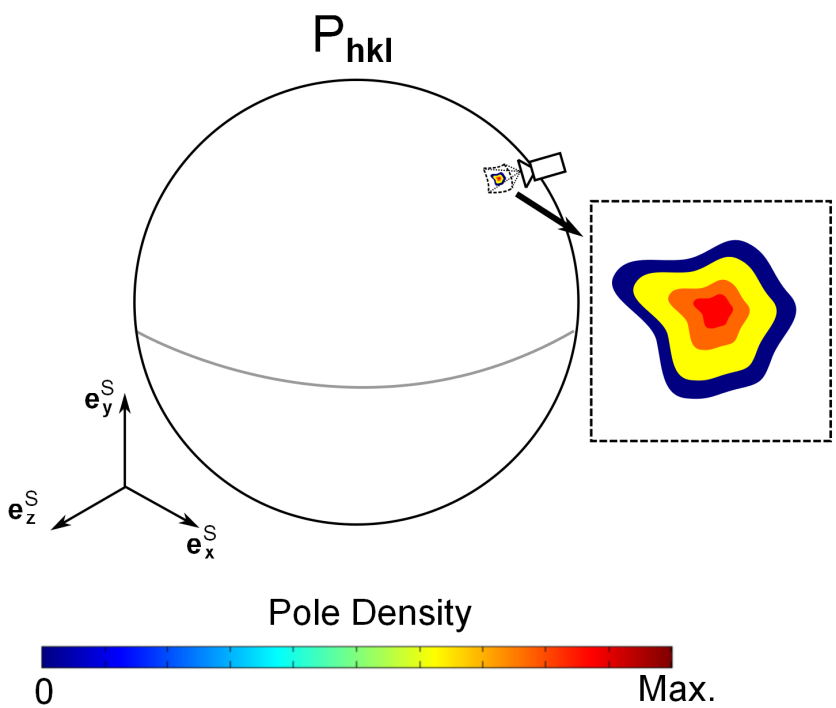

Figure 1: An example orientation pole figure measured from a single crystal (SCPF). The nonzero regions of the distribution are shown from 'aerial view' of the surface. 


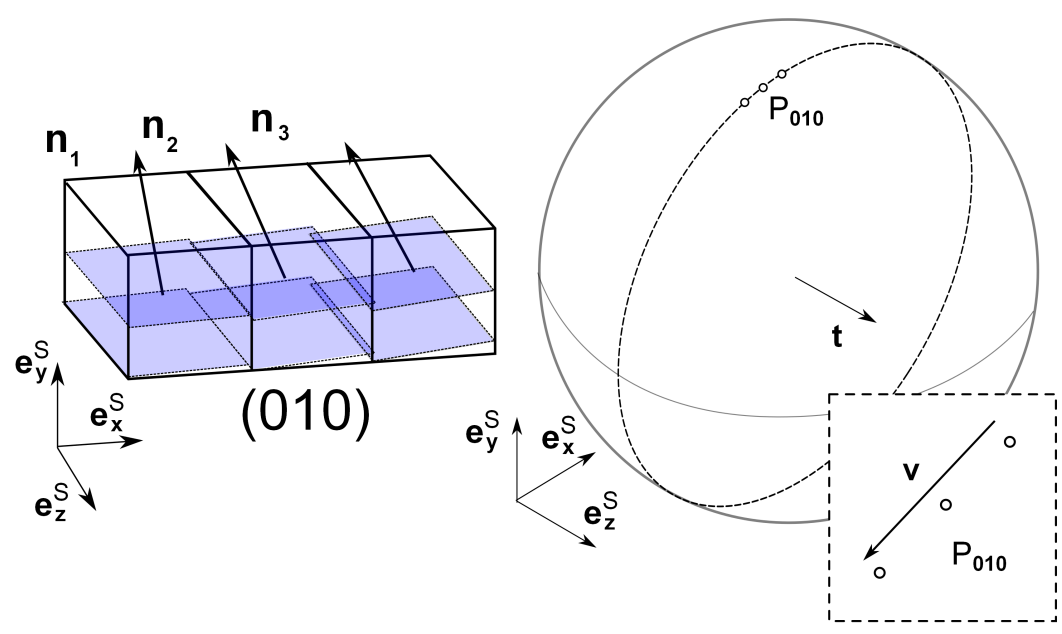

Figure 2: Three volumes of crystal misoriented about the axis $\boldsymbol{t}$ (the [001] crystallographic direction). The (010) lattice planes misoriented about the axis $\boldsymbol{t}$. To the right is the $P_{010}$ single crystal pole figure. The extensions along the dotted trajectory are labeled with the vector $\boldsymbol{v}$. 


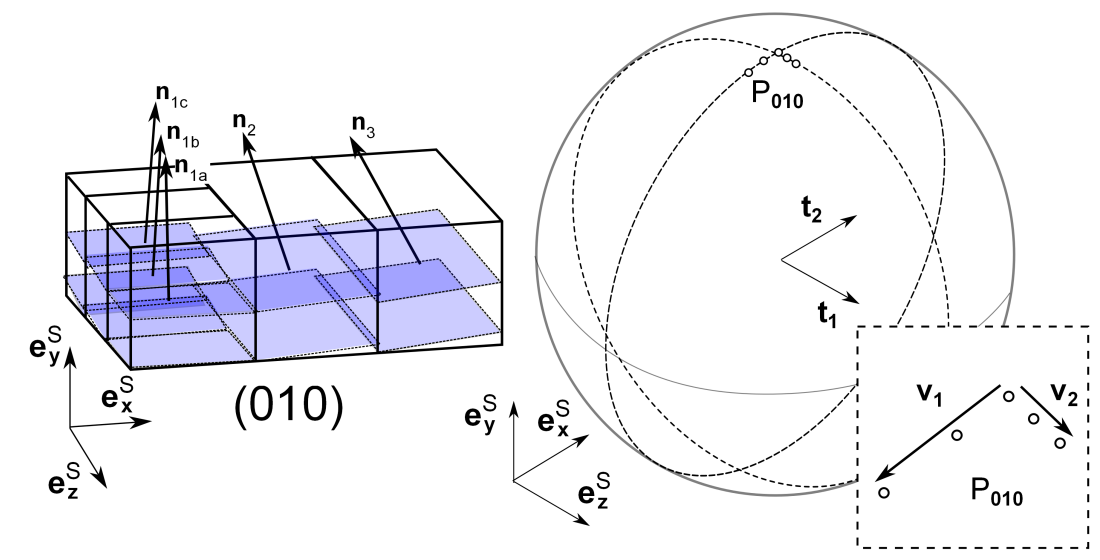

Figure 3: An example of a misorientation configuration of the (010) lattice planes within a crystal that has two distinct misorientation axes, $\boldsymbol{t}_{\mathbf{1}}$ and $\boldsymbol{t}_{\mathbf{2}}$. The $P_{010}$ from the crystal is shown to the right. The pole figure has extended along two distinct trajectories characterized by $\boldsymbol{v}_{\mathbf{1}}$ and $\boldsymbol{v}_{\mathbf{2}}$. 

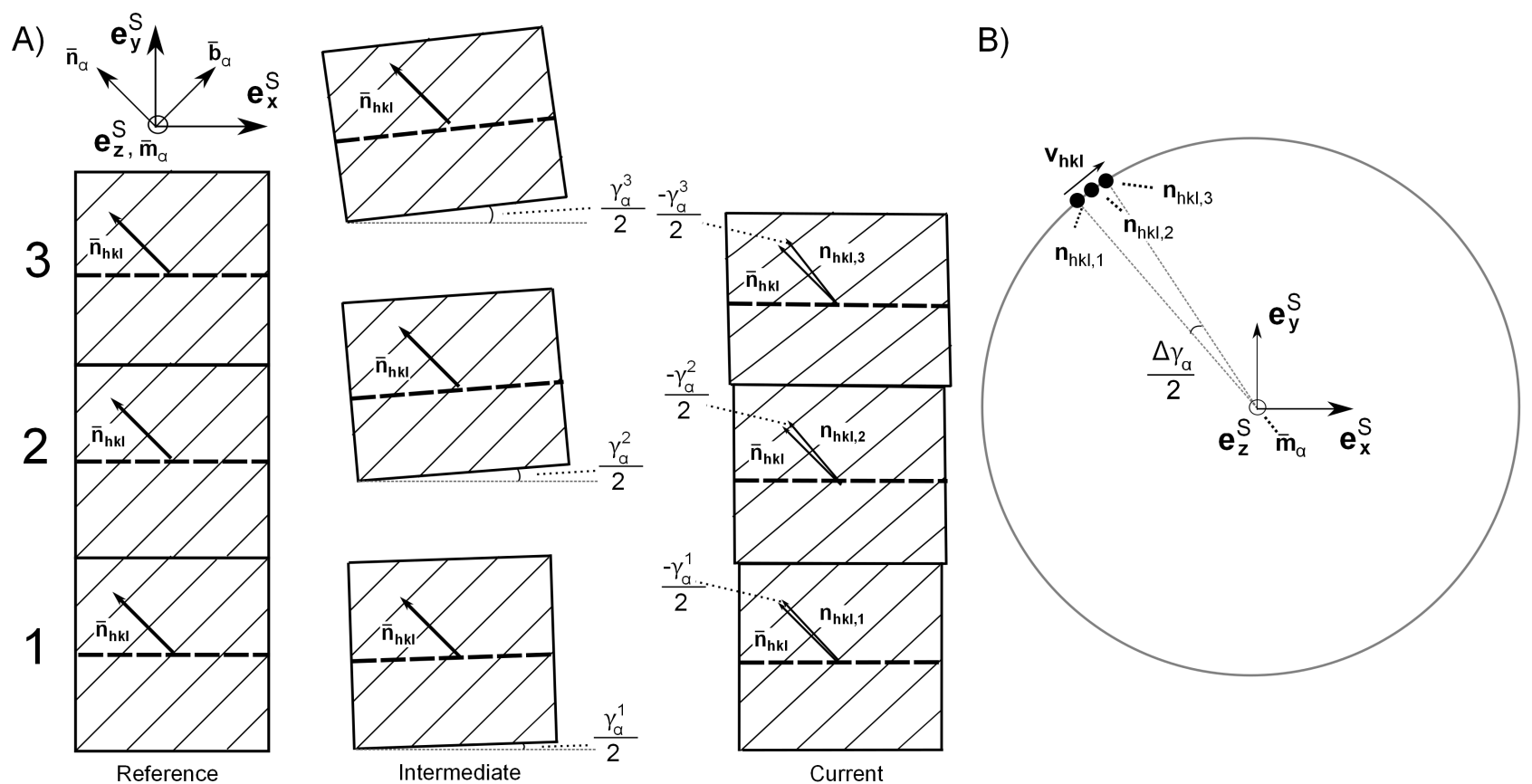

Figure 4: A) Three volumes of a single crystal, initially with the same orientation, shear by varying amounts during compression along the $\boldsymbol{e}_{\boldsymbol{y}}^{S}$ direction. The lattice planes with initial normal $\overline{\boldsymbol{n}}$ are drawn and material planes perpendicular to the compression direction are marked with dashed lines. The volumes are shown in the reference configuration, an intermediate configuration, and the current configuration. B) The current plane normals from (A) are plotted on a pole figure and have spread along $\boldsymbol{v}$. The angle spanned by the distribution of poles is $\frac{\Delta \gamma_{\alpha}}{2}$, where $\Delta \gamma_{\alpha}$ is the max difference in shear strain across the volumes (in this case $\gamma_{\alpha, 3}-\gamma_{\alpha, 1}$ ). 


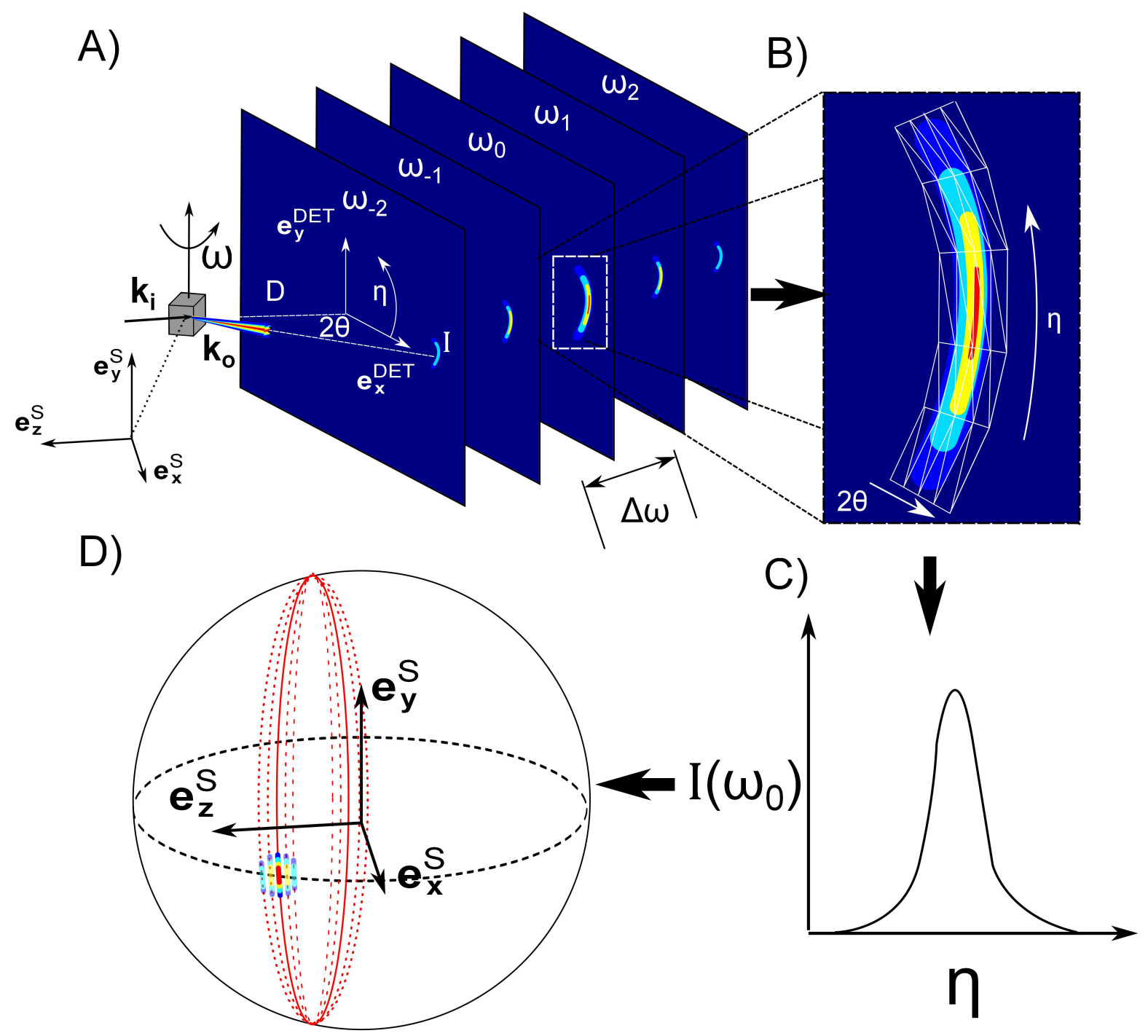

Figure 5: A) A rotating crystal experiment is performed to collect all intensity from a set of lattice planes. The incoming and diffracted wave vectors are $\boldsymbol{k}_{\boldsymbol{i}}$ and $\boldsymbol{k}_{\boldsymbol{o}}$, the sample to detector distance is $D$, diffracted intensity is emitted at the angles $2 \theta$ and $\eta$, and the angle about the vertical axis that the sample is rotated is $\omega$. B) A finite element grid is laid over the raw diffracted intensity to integrate the distribution in the $2 \theta$ direction. C) After integration, diffracted intensity is expressed only in terms of $\eta$ and $\omega$. D) The two dimensional intensity distributions are mapped to the unit sphere to generate a single crystal pole figure. 


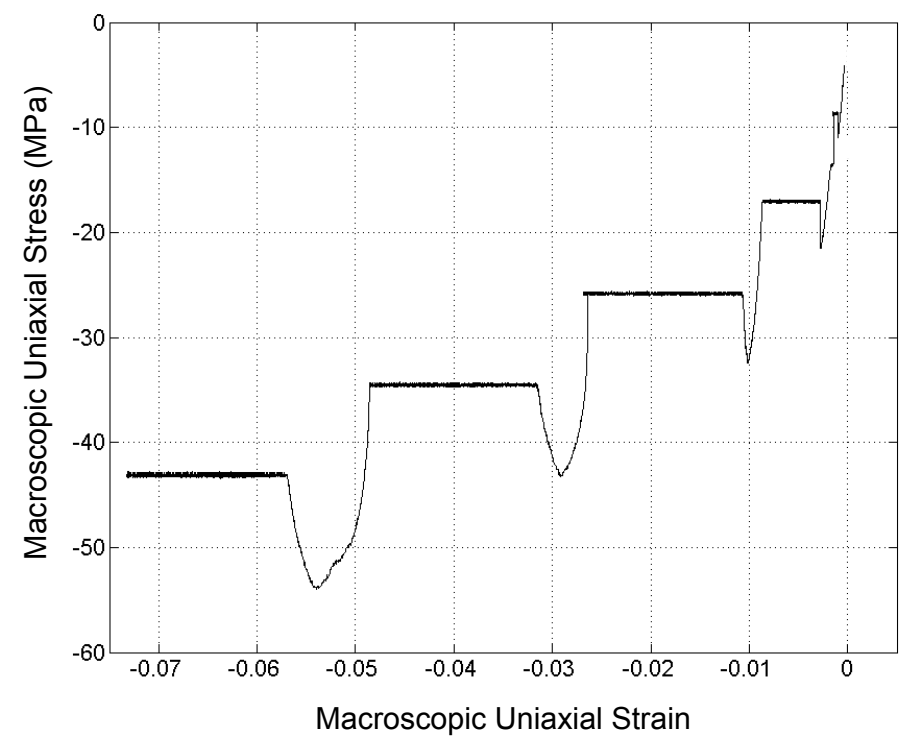

Figure 6: Macroscopic stress-strain data for the silicon compression experiment. 


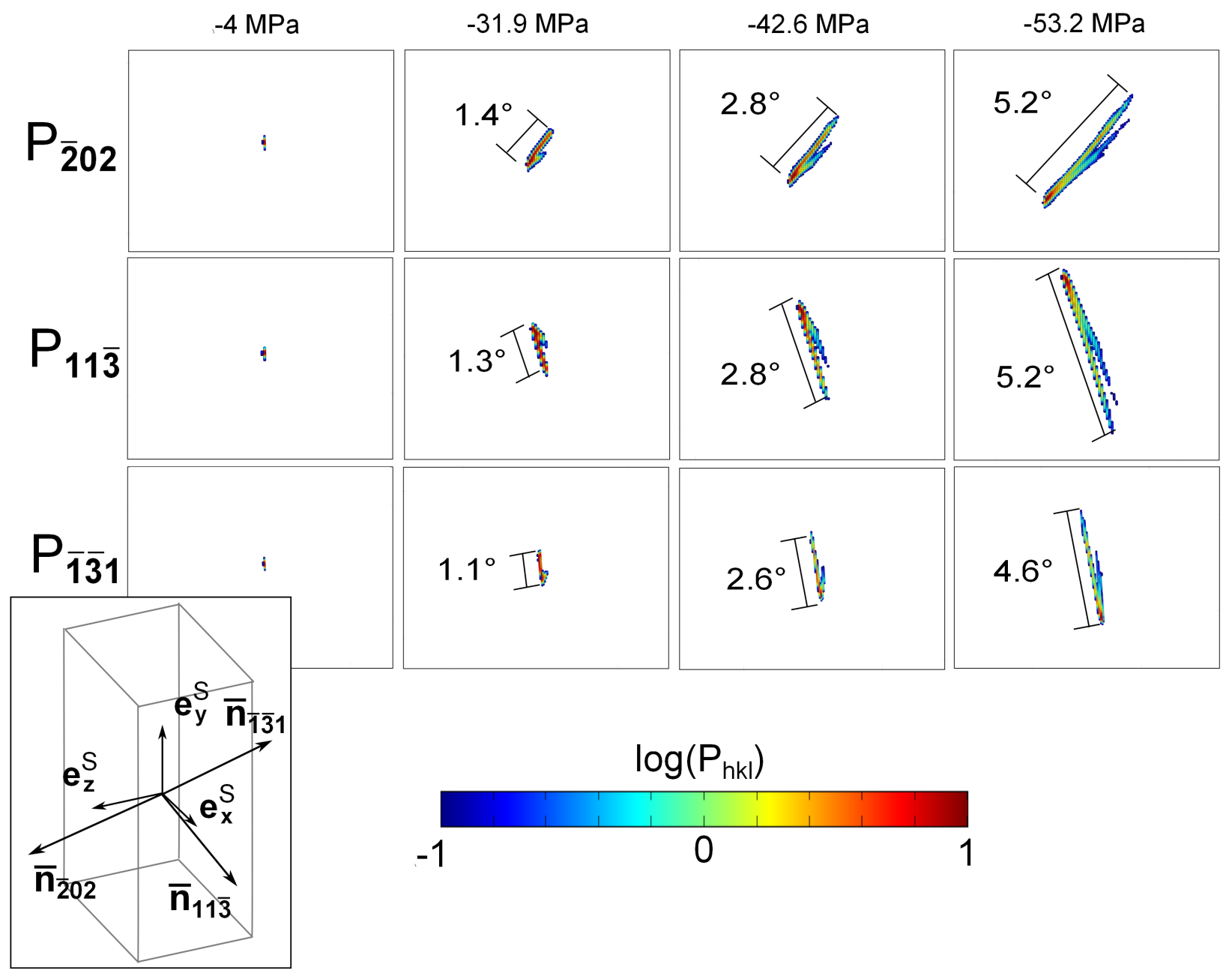

Figure 7: The evolution of three measured single crystal orientation pole figures identified by their Miller indicies $\left(P_{h k l}\right)$ are shown. The measured $P_{h k l}$ have been normalized and are plotted on a logarithmic scale. Only $P_{h k l}$ values above $1 \%$ of the maximum value of each SCPF are shown. The $P_{h k l}$ are shown when the crystal is undeformed and after macroscopic uniaxial stresses of $-31.9,-42.6$, and $-53.2 \mathrm{MPa}$ were applied. The initial positions of the lattice plane normals $\overline{\boldsymbol{n}}$ with respect to the sample are shown. 

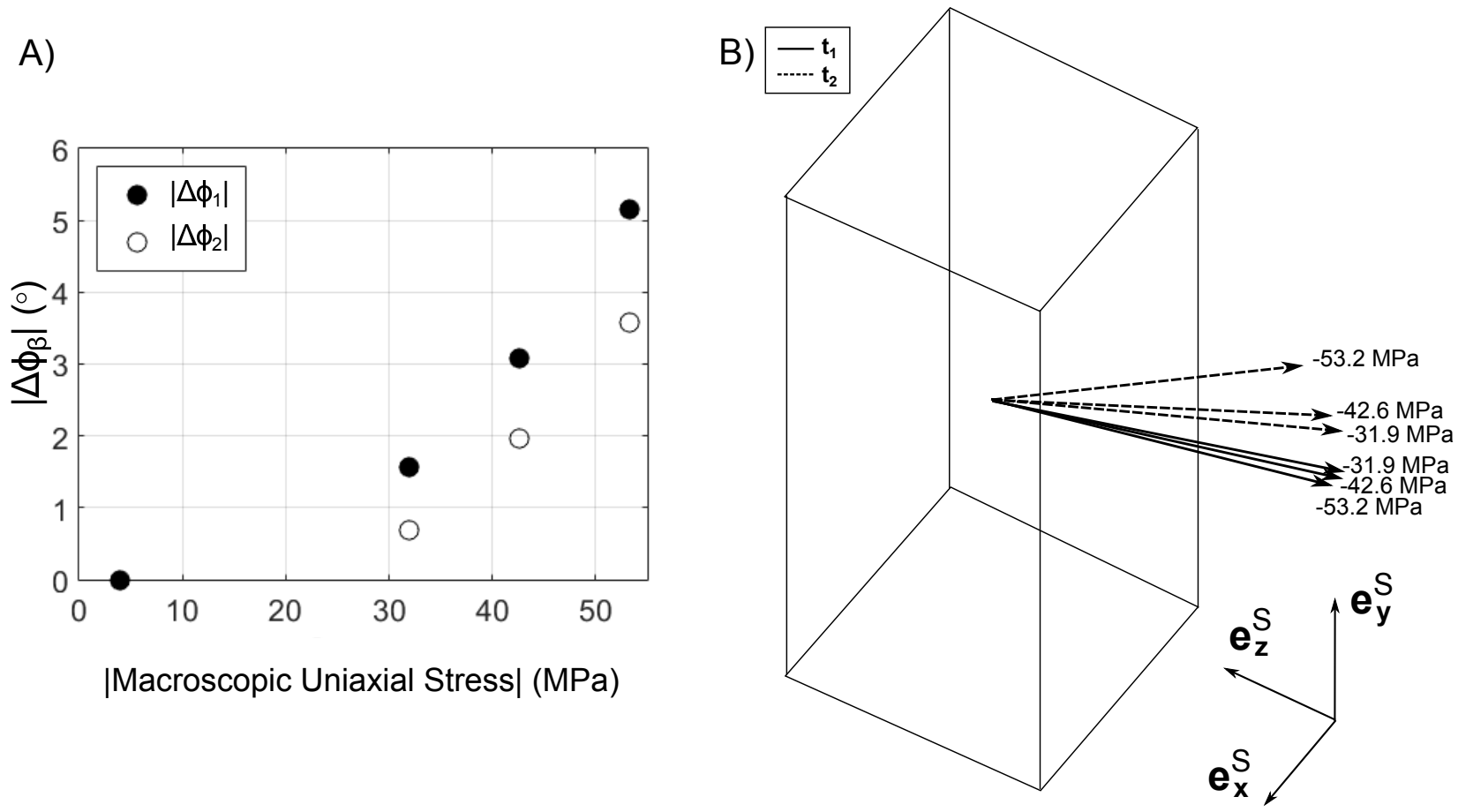

Figure 8: A) Absolute values of the measured $\Delta \phi_{\beta}$ determined from the experimental data with increasing macroscopic load magnitude. Experimental uncertainty in $\Delta \phi_{\beta}$ measurements are $\pm 0.0625^{\circ}$. B) Measured $\boldsymbol{t}_{\boldsymbol{\beta}}$ with respect to the sample also determined from experimental data. 


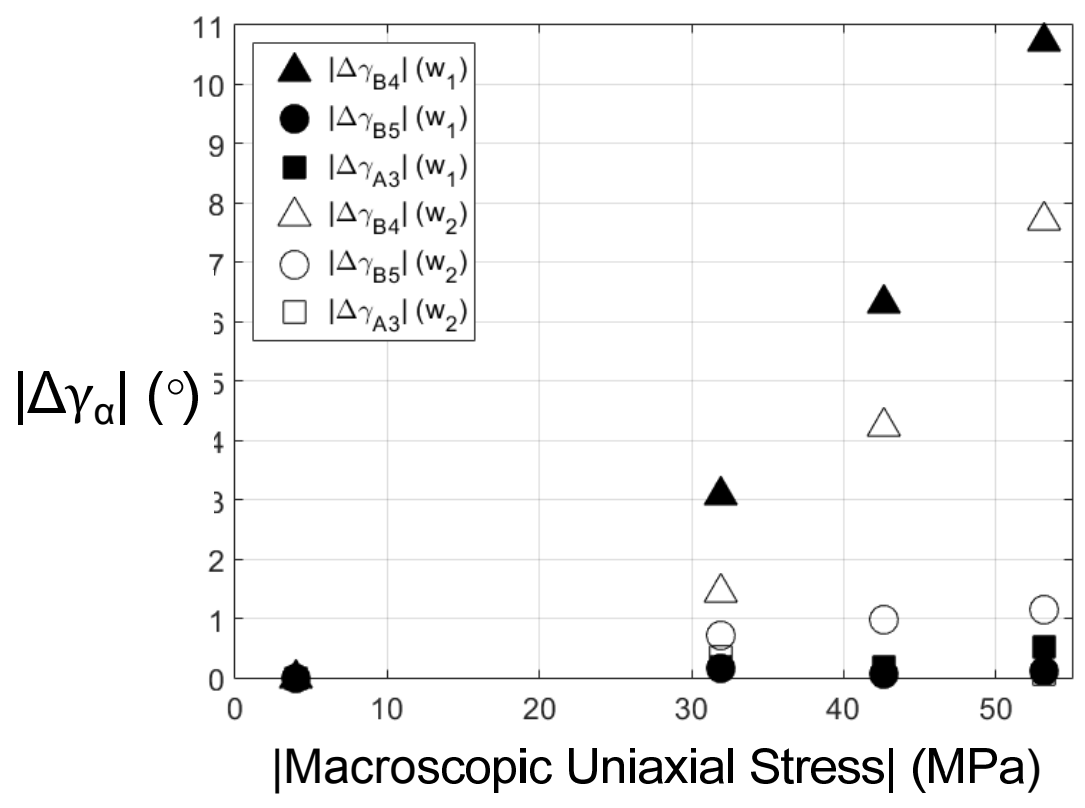

Figure 9: The absolute value of $\Delta \gamma_{\alpha}$ on slip systems B4, B5, and A3 for the final 3 load steps in the experiment. The filled markers are associated with the misorientation vector $\boldsymbol{w}_{\mathbf{1}}$, while the open markers are associated with $\boldsymbol{w}_{\mathbf{2}}$. Experimental uncertainty in $\Delta \gamma_{\alpha}$ measurements are $\pm 0.125^{\circ}$. 


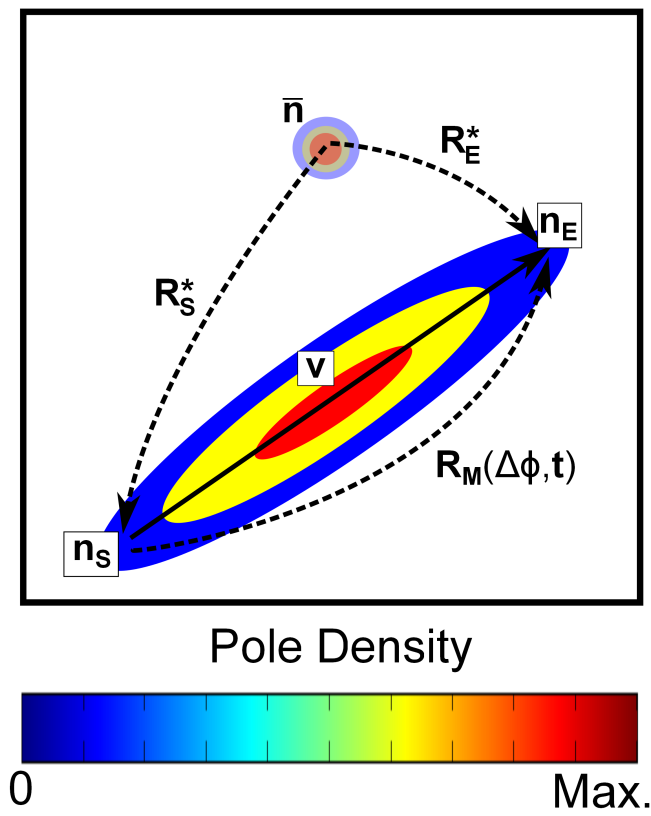

Figure 10: A diagram of the evolution of a single crystal orientation pole figure. The initial plane normal position is $\overline{\boldsymbol{n}}$. After deformation, the plane normal (pole) distribution has spread along the direction $\boldsymbol{v}$. The start and end points of $\boldsymbol{v}$ are $\boldsymbol{n}_{S}$ and $\boldsymbol{n}_{\boldsymbol{E}}$. The rotation $\boldsymbol{R}_{\boldsymbol{M}}$ rotates $\boldsymbol{n}_{\boldsymbol{S}}$ into $\boldsymbol{n}_{\boldsymbol{E}}$ by the angle $\Delta \phi$ about the axis $\boldsymbol{t}$. The rotations $\boldsymbol{R}_{\boldsymbol{S}}^{*}$ and $\boldsymbol{R}_{\boldsymbol{E}}^{*}$ rotate $\overline{\boldsymbol{n}}$ into $\boldsymbol{n}_{\boldsymbol{S}}$ and $\boldsymbol{n}_{\boldsymbol{E}}$ respectively. 


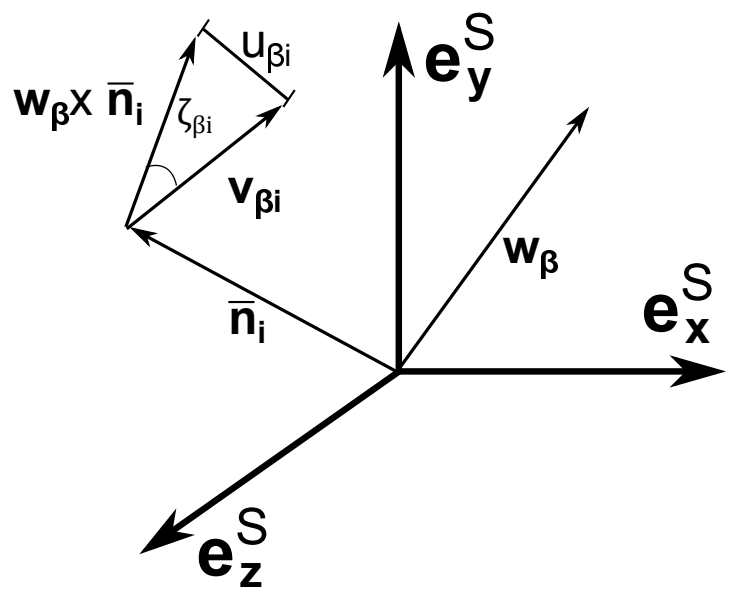

Figure 11: Illustration of the distance $u_{i}$ and angle $\zeta_{i}$ which are minimized in Equation 22 in order to determine the misorientation vectors $\boldsymbol{w}_{\boldsymbol{\beta}}$ from experimentally measured trajectories $\boldsymbol{v}_{\boldsymbol{\beta} i}$ and initial plane normal positions $\overline{\boldsymbol{n}}_{\boldsymbol{i}}$ on single crystal pole figures. 


\begin{tabular}{|c|c|c|c|}
\hline$\alpha$ & $\overline{\boldsymbol{s}}$ & $\overline{\boldsymbol{n}}$ & SF \\
\hline B4 & $\frac{1}{\sqrt{2}}[101]$ & $\frac{1}{\sqrt{3}}[11 \overline{1}]$ & 0.47 \\
\hline B5 & $\frac{1}{\sqrt{2}}[011]$ & $\frac{1}{\sqrt{3}}[11 \overline{1}]$ & 0.35 \\
\hline B2 & $\frac{1}{\sqrt{2}}[1 \overline{1} 0]$ & $\frac{1}{\sqrt{3}}[11 \overline{1}]$ & 0.12 \\
\hline C1 & $\frac{1}{\sqrt{2}}[110]$ & $\frac{1}{\sqrt{3}}[1 \overline{1} 1]$ & 0.29 \\
\hline C5 & $\frac{1}{\sqrt{2}}[011]$ & $\frac{1}{\sqrt{3}}[1 \overline{1} 1]$ & 0.18 \\
\hline C3 & $\frac{1}{\sqrt{2}}[10 \overline{1}]$ & $\frac{1}{\sqrt{3}}[1 \overline{1}]$ & 0.12 \\
\hline D4 & $\frac{1}{\sqrt{2}}[101]$ & $\frac{1}{\sqrt{3}}[1 \overline{1} \overline{1}]$ & 0 \\
\hline D1 & $\frac{1}{\sqrt{2}}[110]$ & $\frac{1}{\sqrt{3}}[1 \overline{1}]$ & 0 \\
\hline D6 & $\frac{1}{\sqrt{2}}[011]$ & $\frac{1}{\sqrt{3}}[1 \overline{1} \overline{1}]$ & 0 \\
\hline A3 & $\frac{1}{\sqrt{2}}[10 \overline{1}]$ & $\frac{1}{\sqrt{3}}[111]$ & 0.35 \\
\hline A6 & $\frac{1}{\sqrt{2}}[0 \overline{1} 1]$ & $\frac{1}{\sqrt{3}}[111]$ & 0.18 \\
\hline A2 & $\frac{1}{\sqrt{2}}[110]$ & $\frac{1}{\sqrt{3}}[111]$ & 0.18 \\
\hline
\end{tabular}

Table 1: Reference slip directions $(\overline{\boldsymbol{s}})$ slip plane normals $(\overline{\boldsymbol{n}})$ expressed in the crystal coordinate system. Also given are the Schmid factors (SF) of the twelve primary slip systems of the silicon single crystal specimen with the [123] crystallographic direction along the loading axis. 


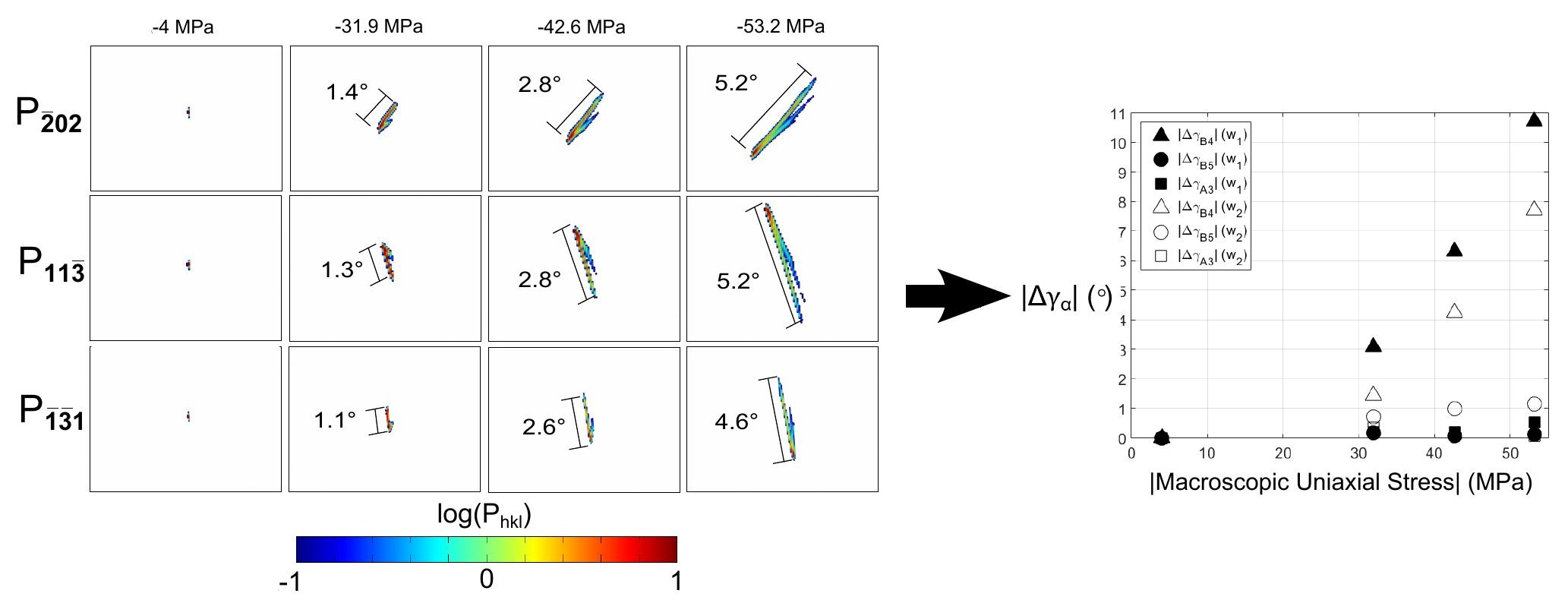

